\title{
The Expression Level of HIV-1 Vif Is Optimized by Nucleotide Changes in the Genomic SA1D2prox Region during the Viral Adaptation Process
}

\author{
Takaaki Koma ${ }^{1}{ }^{1}$, Naoya Doi ${ }^{1}$, Mai Takemoto ${ }^{1,2}$, Kyosuke Watanabe ${ }^{1,2}$, Hideki Yamamoto ${ }^{1,2}$, \\ Satoshi Nakashima ${ }^{1,2}$, Akio Adachi ${ }^{3, *}$ and Masako Nomaguchi ${ }^{1, *}$ \\ 1 Department of Microbiology, Tokushima University Graduate School of Biomedical Sciences, \\ Tokushima 770-8503, Japan; tkoma@tokushima-u.ac.jp (T.K.); naoya@tokushima-u.ac.jp (N.D.); \\ maimaitakemoto@gmail.com (M.T.); watanabekyousuke8888@gmail.com (K.W.); \\ johou.hideki.pokopoko@gmail.com (H.Y.); sterling.bertland@gmail.com (S.N.) \\ 2 Faculty of Medicine, Tokushima University, Tokushima 770-8503, Japan \\ 3 Department of Microbiology, Kansai Medical University, Osaka 573-1010, Japan \\ * Correspondence: adachiak@hirakata.kmu.ac.jp (A.A.); nomaguchi@tokushima-u.ac.jp (M.N.)
}

\section{check for}

updates

Citation: Koma, T.; Doi, N.; Takemoto, M.; Watanabe, K.; Yamamoto, H.; Nakashima, S.; Adachi, A.; Nomaguchi, M. The Expression Level of HIV-1 Vif Is Optimized by Nucleotide Changes in the Genomic SA1D2prox Region during the Viral Adaptation Process. Viruses 2021, 13, 2079. https:/ / doi.org/10.3390/v13102079

Academic Editor: Ester Ballana Guix

Received: 21 July 2021

Accepted: 13 October 2021

Published: 15 October 2021

Publisher's Note: MDPI stays neutral with regard to jurisdictional claims in published maps and institutional affiliations.

Copyright: (c) 2021 by the authors. Licensee MDPI, Basel, Switzerland. This article is an open access article distributed under the terms and conditions of the Creative Commons Attribution (CC BY) license (https:// creativecommons.org/licenses/by/ $4.0 /)$.
Abstract: HIV-1 Vif plays an essential role in viral replication by antagonizing anti-viral cellular restriction factors, a family of APOBEC3 proteins. We have previously shown that naturally-occurring single-nucleotide mutations in the SA1D2prox region, which surrounds the splicing acceptor 1 and splicing donor 2 sites of the HIV-1 genome, dramatically alter the Vif expression level, resulting in variants with low or excessive Vif expression. In this study, we investigated how these HIV-1 variants with poor replication ability adapt and evolve under the pressure of APOBEC 3 proteins. Adapted clones obtained through adaptation experiments exhibited an altered replication ability and Vif expression level compared to each parental clone. While various mutations were present throughout the viral genome, all replication-competent adapted clones with altered Vif expression levels were found to bear them within SA1D2prox, without exception. Indeed, the mutations identified within SA1D2prox were responsible for changes in the Vif expression levels and altered the splicing pattern. Moreover, for samples collected from HIV-1-infected patients, we showed that the nucleotide sequences of SA1D2prox can be chronologically changed and concomitantly affect the Vif expression levels. Taken together, these results demonstrated the importance of the SA1D2prox nucleotide sequence for modulating the Vif expression level during HIV-1 replication and adaptation.

Keywords: HIV-1; Vif expression; SA1D2prox; nucleotide sequence; adaptation; splicing

\section{Introduction}

The HIV-1 Vif protein antagonizes host intrinsic restriction factors APOBEC3 (A3) proteins (A3DE, A3F, A3G, and A3H haplotype II/V /VII), which are cytidine deaminases [1-7]. Among the A3 proteins, A3G displays the strongest antiviral activity. In the absence of Vif, A3G proteins are incorporated into virions and inhibit HIV-1 replication mainly by introducing lethal G-to-A hypermutations into the viral genome in a deaminase-dependent manner [1-8]. Deaminase-independent restriction by A3G to the HIV-1 replication process, reverse transcription and integration, has been also demonstrated [4-7]. Vif works as an adaptor to recruit A3 proteins into the E3 ubiquitin ligase complex and leads to proteasome degradation to evade from their restriction [1-8]. Vif also inhibits transcription, translation, and packaging of A3G in a degradation-independent manner [8]. Once a small number of A3G molecules are incorporated into virions, A3G exerts potent antiviral effects $[9,10]$. Thus, the ability of Vif to counteract A3G is essential for HIV-1 replication especially in A3 proteins-expressing cells such as CD4+ T cells and macrophages.

HIV-1 mRNA species are produced through alternative splicing to express various viral proteins [11-13]. Although a variety of HIV-1 mRNA species are produced by the 
combination of splicing donors (SD1 to SD4) and splicing acceptors (SA1 to SA7), vif mRNA is generated by utilizing SD1 and SA1 sites (Figure 1A) [11-14]. Generally, splicing events are accomplished by an interplay between various host proteins and RNA secondary structures/cis-acting sequences in transcripts [15-17]. Cis-acting sequences, called splicing regulatory elements (SREs), are divided into two groups, enhancers and silencers, and recruit sequence-specific RNA binding factors [15-17]. SREs that are involved in vif transcript production and/or exon2 (the fragment from SA1 to SD2) inclusion have also been found within the upstream region of the vif start codon in the HIV-1 genome (Figure 1B) [18-21], (for review, see [16]). The HIV-1 alternative splicing process usually follows the cellular pre-mRNA splicing mechanism. However, unlike cellular mRNA production in which introns are completely removed, HIV-1 must maintain the full-length of RNA that is packaged into the progeny virion as the genome RNA and is transcribed into Gag/Gag-Pol proteins. Furthermore, well-balanced alternative splicing to ensure optimal HIV-1 mRNA production is also required for viral protein expression. The disruption of this process adversely affects HIV-1 replication. Thus, understanding the mechanism of the regulation of HIV-1 mRNA production is important for the control of viral replication [16,17].

HIV-1 has a high ability to adapt to various environments by acquiring mutations through the high mutation rate of reverse transcriptase (RT) and recombination of viral genomes [22,23]. The highly mutable nature of HIV-1 leads to the generation of various mutants that exhibit drug resistance, evasion from the host immune response, and transmission to new hosts [24-27]. In cases of drug-resistant mutants, they do not always acquire mutations in the sites targeted by drugs. For example, the development of resistance against a protease inhibitor (PI) has been reported to result from the emergence of mutations in the Gag or Envelope (Env) region but not in the Pol-protease region [28-32]. Even in Vif-null HIV-1 clones, adaptive clones that emerged during an adaptation experiment developed A3G resistance without Vif expression [33,34], although there is a report that a Vif-null HIV-1 clone failed to do so [35]. For a Vif-null HIV-1 adaptation, acquisition of mutations in Env has been shown to affect the Gag-Pol packaging and result in the incorporation of a high level of RT into virions, thereby enabling an escape from A3G restriction [33]. It has also been reported in another adaptation experiment that single-nucleotide mutations upstream of the Gag start codon increase virion production, resulting in dilution of the A3G-suppressive effects [34]. Adaptation experiments under restrictive conditions are an effective means to newly identify the site(s)/region(s) involved in evasion from the restriction and to understand a viral adaptation pathway and its mechanism.

Based on comparative sequence analyses of adaptive mutations that emerged in our HIV-1 adaptation system [36], we have identified a number of naturally-occurring single nucleotide mutations (nSNM) that vary the Vif expression levels within a region around the SA1 and SD2 sites located within the pol-integrase sequence, named the SA1D2prox region (Figure 1A). These nSNMs within SA1D2prox that alter the Vif expression levels also affect the replication potential depending on both their Vif expression levels and cellular A3G expression levels [37-40]. Pro-viral clones carrying nSNM are grouped into low-, high-, and excessive-Vif types, which express low, high, and extremely high levels of Vif, respectively. Of these, low- and excessive-Vif types exhibit a decreased growth ability compared to that of a wild-type (WT) clone in cells expressing a high level of A3G [38]. In this study, through adaptation experiments of the low- and excessive-Vif type HIV-1 clones under the A3G restrictive condition, we aimed to characterize the viral adaptation process, especially focusing on identifying novel site(s)/region(s) that affect the Vif expression level. To clarify the relationship between SA1D2prox and Vif expression during in vivo replication, we analyzed the effect of changes in the nucleotide sequence within SA1D2prox on the alteration in Vif expression levels using clinical samples obtained in chronological order. The findings in the present study highlight the importance of the SA1D2prox genomic sequence in determining the Vif expression levels. 
A

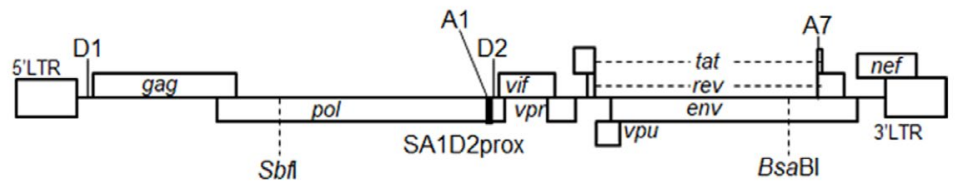

B SA1D2prox

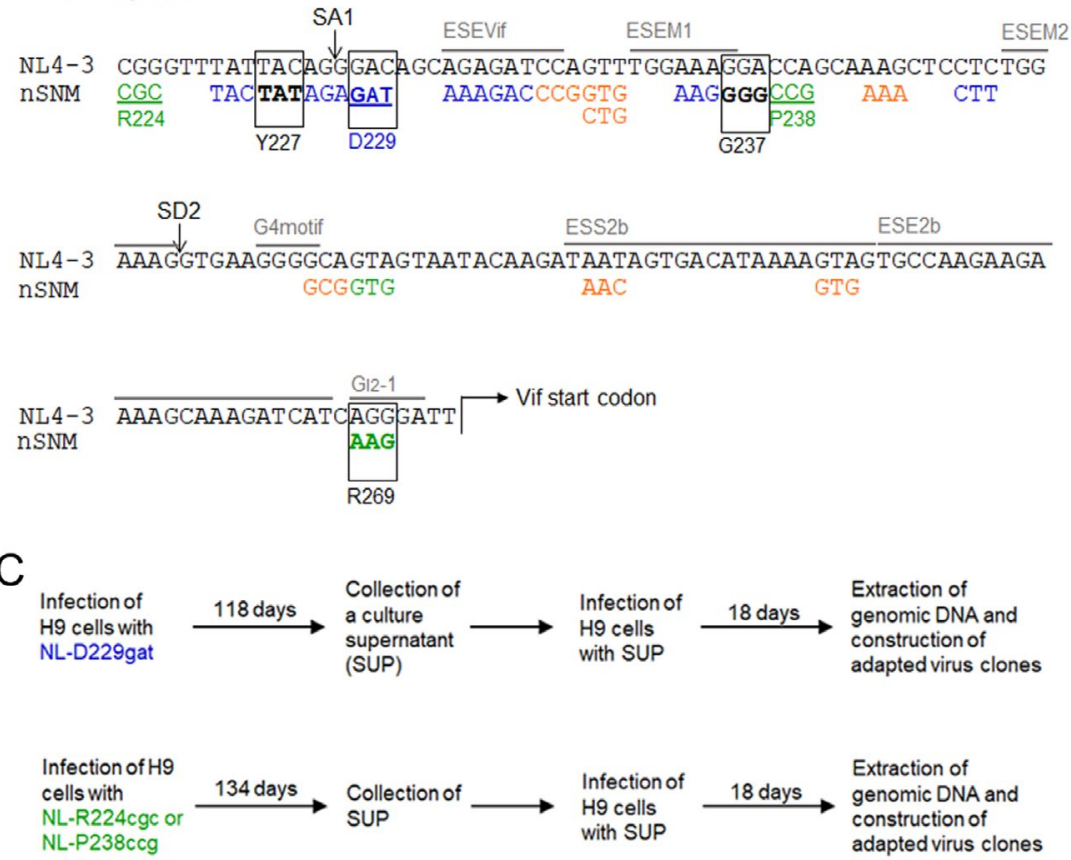

Figure 1. SA1D2prox sequence on the HIV-1 genome and virus adaptation procedure. (A) HIV-1 (NL4-3) genome organization. The SA1D2prox region is shown by a black box. Some of the splicing sites (SD1, SA1, SD2, and SA7) of the HIV-1 genome are indicated. Unique restriction enzyme sites

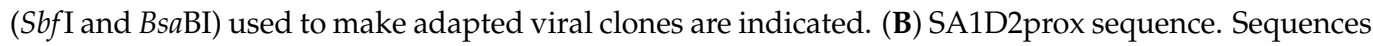
of low (NL-D229gat) and excessive (NL-R224cgc and NL-P238ccg) Vif types used for the adaptation experiments in this study are shown by blue and green letters/lines, respectively. Blue, orange, and green letters without underlines indicate single-nucleotide mutants identified by us [37-39] which display low, high, and excessive Vif types, respectively. Boxed, bold letters show adaptive mutations that change the Vif expression levels identified in this study. Splicing sites (SA1 and SD2) are indicated. Several known splicing enhancer/silencer motifs are represented by gray letters and lines [18-21], (for review, see [16]). nSNM, naturally-occurring single nucleotide mutation. (C) Virus adaptation. Viral clones of low (NL-D229gat) and excessive (NL-R224cgc and NL-P238ccg) Vif types were inoculated into $\mathrm{H} 9$ cells and cultured for the indicated periods. Fresh $\mathrm{H} 9$ cells were infected with the SUP collected on day 118 for NL-D229gat and day 134 for NLR224cgc/NL-P238ccg. On day 18 post-infection, cellular genomic DNA was extracted and used for the construction of adapted viral clones.

\section{Materials and Methods}

\subsection{Plasmids}

Pro-viral clones (WT NL4-3, NL- $\Delta$ Vif clone NL-Nd, NL-D229gat, NL-R224cgc, and NL-P238ccg) and a FLAG-tagged A3G expression vector have been described previously [38,41-44]. To construct the pcNLmini-RI clone, the following fragments were fused by overlapping PCR: fragment 1 (NL4-3 nucleotide positions 675 to 804 including the $\mathrm{XbaI}$ site at the $5^{\prime}$ end) and fragment 2 (NL4-3 nucleotide positions 4842 to 5748 including the authentic EcoRI site at the $3^{\prime}$ end), and then the resultant fragment was introduced into pcDNA3.1(-) vector (Invitrogen). Mutant clones for NL4-3 and for pcNLmini-RI were generated by site-directed mutagenesis. 


\subsection{Cells}

A human embryonic kidney cell line, HEK 293T [45], was cultured in minimal essential medium supplemented with $10 \%$ heat-inactivated fetal bovine serum. A human lymphocyte cell line, H9, was cultured in RPMI 1640 supplemented with 10\% heat-inactivated fetal bovine serum.

\subsection{Adaptation Experiments}

The adaptation experiments performed are outlined in Figure 1. Virus stocks were prepared from HEK 293T cells transfected with pro-viral clones by the calcium phosphate coprecipitation method [41,46]. The virion-associated RT activity was measured as previously described $[47,48]$. Briefly, culture supernatant was mixed with an RT reaction mixture containing poly (A) as a template, oligo $(\mathrm{dT})_{18}$ as a primer, and $\left[\alpha{ }^{3}{ }^{32} \mathrm{P}\right] \mathrm{dTTP}$. After incubation, the reaction mixture was spotted onto a positively charged nylon membrane (Roche). The membrane was washed with $2 \times$ SSC to remove unincorporated $\left[\alpha-{ }^{32} \mathrm{P}\right] \mathrm{dTTP}$, and spots were then counted by scintillation counter. Equal units of RT activity $\left(10^{4}\right)$ were inoculated into $\mathrm{H} 9$ cells $\left(10^{6}\right)$ for the adaptation experiments of NL-D229gat, NL-R224cgc, and NL-P238ccg. The long-term culture of infected cells was carried similarly to that described previously [36]. Fresh $\mathrm{H} 9$ cells were added to the culture twice and three times for NL-D229gat and NL-R224cgc/NL-P238ccg, respectively, during the long-term culture in order to rescue adapted viruses. To construct adapted pro-viral clones, fragments from the $S b f$ I site to the BsaBI site (Figure 1A) were amplified by PCR and introduced into the corresponding sites of the NL4-3 clone. For selected clones, sequence analyses were done for the region from the $S b f \mathrm{I}$ to the $B s a \mathrm{BI}$ sites.

\subsection{Infection Experiments}

Virus stocks were prepared from HEK 293T cells transfected with pro-viral clones by the calcium phosphate coprecipitation method [41,46] or by Lipofectamine2000 (Invitrogen, Waltham, MA, USA). The virion-associated RT activity was measured, and equal RT units were inoculated into $\mathrm{H} 9$ cells for the analysis of viral clones. Virus replication was monitored by the activity of RT released into the culture supernatants.

\subsection{Western Blotting Analysis}

Western blotting analysis was performed as described previously [37,38]. Briefly, pro-viral clones or pcNLmini-RI-based clones were transfected into HEK 293T cells by Lipofectamine2000, and unless otherwise noted, on day 1, post-transfection cell lysates were prepared with $1 \times$ TNE buffer. Analyses of Vif and A3G expression levels in virions and cells were carried out similarly as described previously [43,44]. Briefly, HEK 293T cells were co-transfected with pro-viral clones along with a FLAG-tagged A3G expression vector by the calcium phosphate coprecipitation method. On day 1 post-transfection, virions were collected by ultracentrifugation, and the lysates of virions and cells were prepared with $1 \times$ TNE buffer. Anti-HIV-1 Vif 319 (catalog no. ab66643; Abcam, Tokyo, Japan), anti-Gag-p24 (\#3537, NIH Research and References Reagent Program), anti-FLAG (FLA-1, MEDICAL \& BIOLOGICAL LABORATORIES), and anti- $\beta$-actin clone AC-15 (Sigma-Aldrich, Burlington, MA, USA) antibodies were used for immunoblotting analyses.

\subsection{Semiquantitative RT-PCR Analysis of Splicing Products}

HEK 293T cells were transfected with pcNLmini-RI or its variant clones. As described previously [38,39], at $16 \mathrm{hrs}$ post-transfection, cells were lysed for RNA extraction, and subsequent $\mathrm{cDNA}$ synthesis using oligo (dT) primer. PCR amplification was done similarly as described previously utilizing the following primer sets: XbaI-NL675-5 (Forward) (GCTCTAGAGAGGAGATCTCTCGACGCAG) and NL741-3 (Reverse) (GTCGCCGCCCCTCGCCTC) for all transcripts; XbaI-NL675-5 (Forward) and Vif-qPCR-3 (Reverse) (ACCTGCCATCTGTTTTCCATA) for the full-length and D1/A1 splicing products; XbaI-NL675-5 (Forward) and NL5622-3 (Reverse) (GCTCTAGTGTCCATTCATTG) for the D1/A1-D2/A2 
and D1/A2 splicing products. PCR amplicons were resolved by Metaphor agarose gel (Lonza) and analyzed by the Amersham Imager 600 instrument (GE Healthcare, Chicago, IL, USA) [38].

\section{Results}

\subsection{Viral Clones Used for the Adaptation Experiments}

We have reported various nSNMs within the SA1D2prox region of the HIV-1 (NL4-3) genome that significantly affect the Vif expression level and viral replication potential (Figure 1A) [37-40]. Of these mutant clones, NL-D229gat and NL-R224cgc/NL-P238ccg are categorized into low-Vif and excessive-Vif types, respectively (Figure 1B). To evaluate Vif expression kinetics, HEK 293T cells were transfected with pro-viral clones NL4-3, NL-D229gat, NL-R224cgc or NL-P238ccg. On day 1 and day 2 post-transfection, the cells were lysed and subjected to Western blotting analysis. As shown in Figure 2A, Vif expression levels in NL-D229gat and NL-R224cgc/NL-P238ccg were very low and significantly high, respectively, compared to that in WT NL4-3. The difference in Vif expression levels among these clones was maintained at different time points of posttransfection. We thus decided to carry out experiments using samples prepared on day 1 post-transfection thereafter. To determine the effect of variation in Vif expression level on A3G antagonizing activity, pro-viral clones along with a FLAG-tagged A3G expression vector were co-transfected into HEK $293 \mathrm{~T}$ cells. An NL- $\Delta$ Vif clone was used for the negative control, and a WT NL4-3 clone was used for the positive control. On day 1 post-transfection, the lysates of virions and cells were prepared for Western blotting analysis (Figure 2B). For a negative control NL- $\Delta$ Vif, significant amounts of A3G were observed in virions and cells relative to that for WT NL4-3. The A3G expression levels in virions and cells from low-Vif type NL-D229gat were similar to those in NL- $\Delta$ Vif and significantly higher than those in WT NL4-3 and excessive-Vif type NL-R224cgc/NL-P238ccg (Figure 2B). A3G expression levels in virions and cells from excessive-Vif type NL-R224cgc/NL-P238ccg were similar to those in WT NL4-3, whereas Vif levels for the excessive clones were substantially higher than those for the other clones in cells and virions (Figure 2B). The results in Figure 2 indicate that low-Vif type NL-D229gat is not able to efficiently degrade A3G, and that, while excessive-Vif type NL-R224cgc/NL-P238ccg retain A3G antagonizing activity, large amounts of Vif were incorporated into virions of the two viral clones. A high level of HIV-1 Vif expression has been shown to inhibit viral infectivity [49] and to reduce virion production via the decrease in accumulation of gag-pol mRNA $[19,50]$. Indeed, we have shown that excessive-Vif type NL-R224cgc/NL-P238ccg exhibit reduced virus infectivity and virion production, and thus their viral replication abilities are decreased compared to WT NL4-3 [38]. Thus, the three variant clones grew poorly compared to the WT NL4-3 clone in the highly A3G-expressing H9 cells, which also express A3DE and A3F [38,51]. H9 cells have been reported to express A3G level similar to that in PBMC, though individual differences were observed $[38,52,53]$. In this study, we performed adaptation experiments using the low- and excessive-Vif type viral clones and the potently virus-restrictive H9 cell line as host cells (Figure 1C). To construct adapted pro-viral clones, we utilized the fragment from the $S b f \mathrm{I}$ site to the $B s a \mathrm{BI}$ site of the obtained adapted viruses to maintain a series of SD and SA sites, except for the SD1 and SA7 sites, on the viral genome (Figure 1A). Through analyses of the adapted clones, we specifically explored the sites involved in the alteration of the Vif expression level. 
A

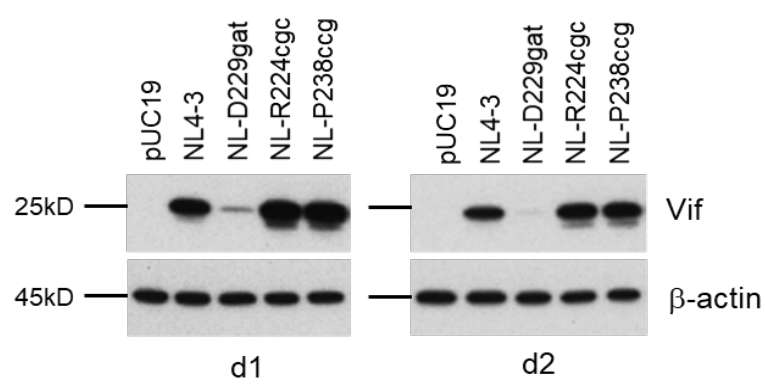

B

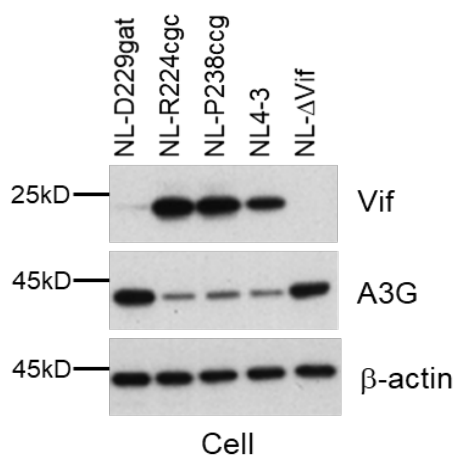

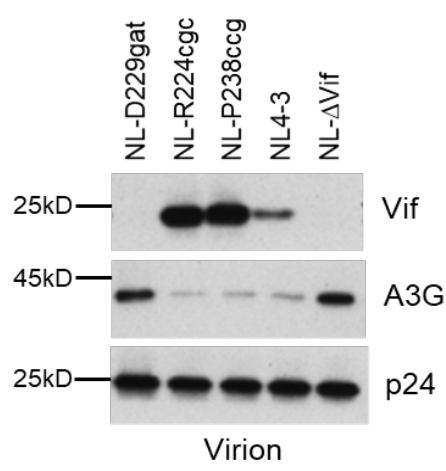

Figure 2. Characteristics of low-Vif type NL-D229gat and excessive-Vif type NL-R224cgc/NL-P238ccg for Vif expression level and A3G degrading activity. (A) Vif expression kinetics. HEK 293T cells were transfected with the indicated pro-viral clones. On day 1 and day 2 (d1 and d2, respectively) post-transfection, cell lysates were prepared and subjected to Western blotting analysis using anti-Vif and anti- $\beta$-actin antibodies. Representative data from two independent experiments are shown. (B) A3G antagonizing activity. The indicated pro-viral clones along with a FLAG-tagged A3G expression vector were co-transfected into HEK $293 \mathrm{~T}$ cells. On day 1 post-transfection, virions and cells were collected and lysed for Western blotting analysis using anti-Vif, anti-Gag-p24, anti-FLAG, and anti- $\beta$-actin antibodies. Representative data from two independent experiments are shown.

\subsection{Characteristics of Viral Clones Obtained from Low-Vif Type NL-D229gat Adaptation}

We performed a long-term culture of NL-D229gat-infected H9 cells as outlined in Figure 1C, and constructed adapted (NL-gatad) clones as described above. To test the replication-competence of NL-gatad clones, a multi-cycle infection experiment was performed (Figure 3A). Viruses prepared from HEK 293T cells transfected with a WT NL4-3, a parental NL-D229gat, or seven NL-gatad clones were inoculated into H9 cells. Virus replication was monitored by RT activity in the culture supernatants collected every 3 days post-infection. As shown in Figure 3A, consistent with our previous result [38], the NLD229gat displayed significantly delayed kinetics relative to that of NL4-3. All the NL-gatad clones tested, except for NL-gatad-5, exhibited higher growth potentials than the parental NL-D229gat, albeit still lower relative to NL4-3. To examine the alteration in the Vif expression levels, these clones were transfected into HEK 293T cells. As shown in Figure 3B, the Vif expression levels of NL-gatad clones 1, 5, 6, and 7 were clearly increased compared to that of NL-D229gat, whereas NL-gatad clones 4, 9, and 10 displayed similar expression levels to that of NL-D229gat. We carried out sequence analysis of several NL-gatad clones (1, 4, 6, 7, and 9). NL-gatad clones 4 and 9, whose Vif expression levels were not altered, carry several mutations in the Vif, Vpu/Env, and Env regions (Table 1). As for NL-gatad-9, while a mutation (D270gac) was found within the SA1D2prox region, we have shown previously that the D270gac mutation does not significantly affect the vif transcript level (0.96 on average relative to NL4-3) [39]. Thus, clones 4 and 9 exhibited enhanced growth potential probably by acquiring mutation(s) without an increase in the Vif expression levels. On the other hand, various mutations are present throughout the genomes of NL-gatad clones 1, 6, and 7, which exhibited the increased Vif expression level (Table 2). Most importantly, all three clones carried the R269Kaag mutation within the SA1D2prox region. This mutation, a natural variation found in the $\mathrm{G}_{\mathrm{I} 2-1}$ element, has been experimentally shown to increase the Vif expression level (Figure 1B) [38]. 
A

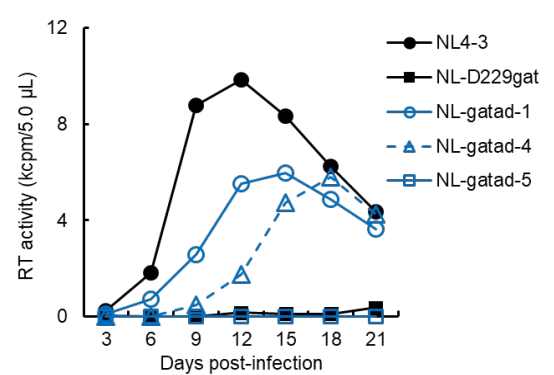

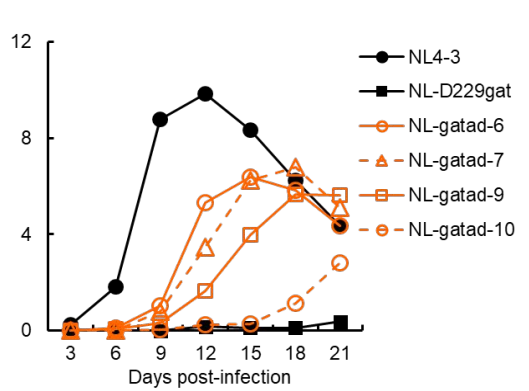

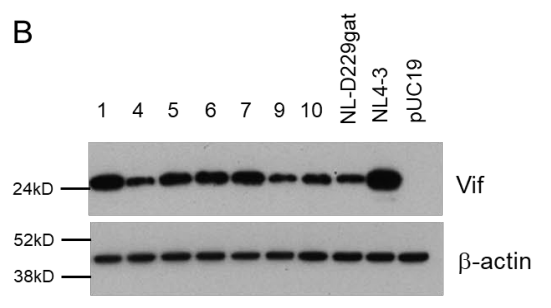

Figure 3. Characteristics of viral clones constructed from adapted NL-D229gat. (A) Growth kinetics. Viruses prepared from HEK 293T cells transfected with the indicated pro-viral clones were inoculated into H9 cells $\left(1 \times 10^{4}\right.$ RT units $/ 10^{5}$ cells). Virus replication was monitored by the virion-associated RT activity in the culture supernatants. This experiment was performed once to select viral clones that have the ability to grow in cells. Viral replication kinetics of WT NL4-3 and a parental NL-D229gat are presented in both panels for easy comparison. (B) Vif expression levels. HEK 293T cells were transfected with the indicated pro-viral clones. On day 1 post-transfection, cell lysates were prepared and subjected to Western blotting analysis using anti-Vif and anti- $\beta$-actin antibodies. An empty vector pUC19 and an authentic HIV-1 NL4-3 clone were used as negative and positive controls, respectively. Numbers correspond to those for the adapted clones shown in panel (A). Representative data from two independent experiments are shown.

Table 1. Mutations found in adapted clones which exhibit similar Vif expression levels to that in the NL-D229gat clone.

\begin{tabular}{cccccc}
\hline & \multicolumn{2}{c}{ NL-gatad-4 } & & \multicolumn{2}{c}{ NL-gatad-9 } \\
Nt Change & Region & NS/S Change in the Region & Nt Change & Region & NS/N Change in the Region \\
\hline \multirow{2}{*}{ c4916t } & $\begin{array}{c}\text { Pol-IN } \\
\text { (SA1D2prox) }\end{array}$ & D229gat & c4916t & $\begin{array}{c}\text { Pol-IN } \\
\text { (SA1D2prox) } \\
\text { Pol-IN }\end{array}$ & D229gat \\
g5389a & & & t5039c & (SA1D2prox) & D270gac \\
g6280a & Vif & & g5389a & Vif & E117K \\
& Vpu & E117K & g6280a & Env & M20I \\
g7016a & Env & A74T & & & \\
g7032a & Env & M20I & & & \\
\hline
\end{tabular}

$\mathrm{Nt}$, Nucleotide; NS, non-synonymous; S, synonymous.

Table 2. Mutations found in adapted clones that exhibit higher Vif expression levels than that in the NL-D229gat clone.

\begin{tabular}{|c|c|c|c|c|c|c|c|c|}
\hline \multicolumn{3}{|c|}{ NL-Gatad-1 } & \multicolumn{3}{|c|}{ NL-Gatad-6 } & \multicolumn{3}{|c|}{ NL-Gatad-7 } \\
\hline $\begin{array}{c}\text { Nt } \\
\text { Change }\end{array}$ & Region & $\begin{array}{l}\text { NS/S Change } \\
\text { in the Region }\end{array}$ & $\begin{array}{c}\text { Nt } \\
\text { Change }\end{array}$ & Region & $\begin{array}{l}\text { NS/N Change } \\
\text { in the Region }\end{array}$ & $\begin{array}{c}\text { Nt } \\
\text { Change }\end{array}$ & Region & $\begin{array}{l}\text { NS/N Change } \\
\text { in the Region }\end{array}$ \\
\hline g3301a & Pol-RT & S251N & g3301a & Pol-RT & S251N & g3301a & Pol-RT & S251N \\
\hline g3341a & Pol-RT & L264tta & g3341a & Pol-RT & L264tta & g3341a & Pol-RT & L264tta \\
\hline \multirow[t]{2}{*}{ g3545a } & Pol-RT & Q332caa & g3545a & Pol-RT & Q332caa & g3545a & Pol-RT & Q332caa \\
\hline & & & & & & $\mathrm{g} 4220 \mathrm{a}$ & Pol-RT & R557aga \\
\hline g4374a & Pol-IN & $\mathrm{A} 49 \mathrm{~T}$ & g4374a & Pol-IN & $\mathrm{A} 49 \mathrm{~T}$ & g4374a & Pol-IN & $\mathrm{A} 49 \mathrm{~T}$ \\
\hline c4916t & $\begin{array}{c}\text { Pol-IN } \\
\text { (SA1D2prox) }\end{array}$ & D229gat & c4916t & $\begin{array}{c}\text { Pol-IN } \\
\text { (SA1D2prox) }\end{array}$ & D229gat & c4916t & $\begin{array}{c}\text { Pol-IN } \\
\text { (SA1D2prox) }\end{array}$ & D229gat \\
\hline g5035a & $\begin{array}{c}\text { Pol-IN } \\
\text { (SA1D2prox) }\end{array}$ & R269Kaag & g5035a & $\begin{array}{c}\text { Pol-IN } \\
\text { (SA1D2prox) }\end{array}$ & R269Kaag & g5035a & $\begin{array}{c}\text { Pol-IN } \\
\text { (SA1D2prox) }\end{array}$ & R269Kaag \\
\hline a5702g & $\mathrm{Vpr}$ & E48gag & $\mathrm{a} 5702 \mathrm{~g}$ & $\mathrm{Vpr}$ & E48gag & a5702g & $\mathrm{Vpr}$ & E48gag \\
\hline g6218a & Vpu & G53D & & & & & & \\
\hline \multirow[t]{4}{*}{ g6280a } & Vpu & A74T & g6280a & Vpu & $\mathrm{A} 74 \mathrm{~T}$ & g6280a & Vpu & A74T \\
\hline & Env & M20I & & Env & M20I & & Env & M20I \\
\hline & & & g6603a & Env & $\mathrm{S} 128 \mathrm{~N}$ & g6603a & Env & $\mathrm{S} 128 \mathrm{~N}$ \\
\hline & & & $\begin{array}{c}\text { 7388- } \\
\text { 7402del }\end{array}$ & Env & $\begin{array}{c}\text { 390-394del } \\
\text { (NSTWFdel) }\end{array}$ & $\begin{array}{c}\text { 7388- } \\
\text { 7402del }\end{array}$ & Env & $\begin{array}{l}\text { 390-394del } \\
\text { (NSTWFdel) }\end{array}$ \\
\hline
\end{tabular}

Bold letters show adaptive mutations that change the Vif expression levels of a parental NL-D229gat clone. Nt, Nucleotide; NS, nonsynonymous; $\mathrm{S}$, synonymous. 


\subsection{Characteristics of Viral Clones Obtained from Excessive-Vif Type NL-R224cgc Adaptation}

We constructed adapted clones (NL-cgcad) derived from a long-term culture of NLR224cgc-infected cells as described above (Figure 1C), and examined their replication abilities along with WT NL4-3 and the parental NL-R224cgc clones (Figure 4A). As we have shown previously [38], NL-R224cgc displayed moderately reduced growth potential relative to NL4-3. The replication ability of three NL-cgcad clones 3, 7, and 10 was comparable to that of WT NL4-3, and NL-cgcad-1 grew better than NL-R224cgc, but to a lesser extent compared to NL4-3 (note the peak days for RT production). As for the growth kinetics of the other clones, NL-cgcad-4 and NL-R224cgc grew similarly, whereas clones 2, 6, and 8 displayed poor growth compared to NL-R224cgc (Figure 4A). Of the eight adapted clones tested, the Vif expression level of clones 1,2, and 4 was markedly reduced to a varying degree relative to those for both NL-R224cgc and NL4-3 (Figure 4B). The sequences of NL-cgcad clones 3, 7, and 10 are identical to that of NL4-3, suggesting a reversion of R224cgc to R224cgg (Figure 1B). NL-cgcad clones 1, 2, and 4, which displayed a remarkably reduced level of Vif expression, carry some mutations (Y227tat, D229gat, and I267att) within the SA1D2prox region (Table 3). Of these mutations, D229gat is the same mutation used in this study (Figures 1 and 3), and the I267att mutation moderately reduced the vif transcript level (0.71 on average relative to NL4-3) [39]. NL-cgcad clones 1, 2, and 4 have many mutations including a Vif deletion, a Vpr deletion, and Env mutations (Table 3). The difference in growth potential among NL-cgcad clones 1, 2, and 4 may be due to the Vif expression level and/or other mutation(s), which can affect the viral replication ability.

A

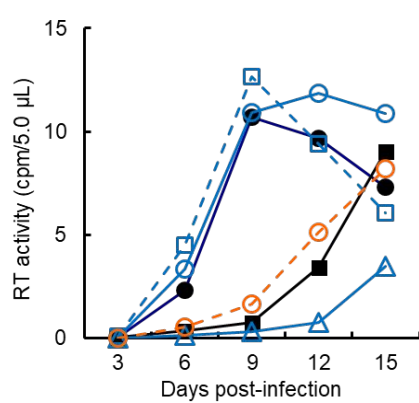

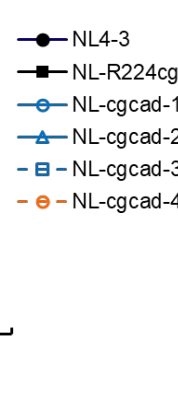
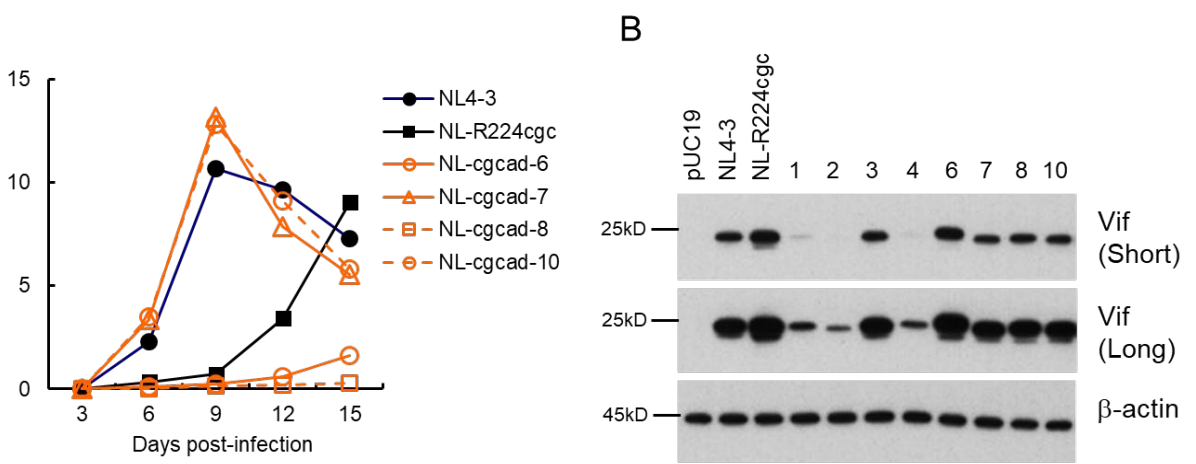

Figure 4. Characteristics of viral clones constructed from adapted NL-R224cgc. (A) Growth kinetics. Viruses prepared from HEK 293 T cells transfected with the indicated pro-viral clones were inoculated into H9 cells ( $2 \times 10^{3}$ RT units $/ 10^{5}$ cells). Virus replication was monitored by the virion-associated RT activity in the culture supernatants. This experiment was performed once to select viral clones that have the ability to grow in cells. Viral replication kinetics of WT NL4-3 and a parental NL-R224cgc are presented in both panels for easy comparison. (B) Vif expression levels. Western blotting analysis was carried out as described in Figure 3B. Numbers correspond to those for the adapted clones shown in panel (A). Representative data from two independent experiments are shown. Short, short exposure; Long, long exposure.

Table 3. Mutations found in adapted clones with decreased Vif expression levels compared to that in the NL-R224cgc clone.

\begin{tabular}{|c|c|c|c|c|c|c|c|c|}
\hline \multicolumn{3}{|c|}{ NL-cgcad-1 } & \multicolumn{3}{|c|}{ NL-cgcad-2 } & \multicolumn{3}{|c|}{ NL-cgcad-4 } \\
\hline $\begin{array}{c}\mathrm{Nt} \\
\text { Change }\end{array}$ & Region & $\begin{array}{l}\text { NS/S Change } \\
\text { in the Region }\end{array}$ & $\begin{array}{c}\text { Nt } \\
\text { Change }\end{array}$ & Region & $\begin{array}{l}\text { NS/N Change } \\
\text { in the Region }\end{array}$ & $\begin{array}{c}\mathrm{Nt} \\
\text { Change }\end{array}$ & Region & $\begin{array}{l}\text { NS/N Change } \\
\text { in the Region }\end{array}$ \\
\hline & & & & & & a3153t & Pol-RT & I202L \\
\hline g4901c & $\begin{array}{c}\text { Pol-IN } \\
\text { (SA1D2prox) }\end{array}$ & $\mathrm{R} 224 \mathrm{cgc}$ & g4901c & $\begin{array}{c}\text { Pol-IN } \\
\text { (SA1D2prox) }\end{array}$ & $\mathrm{R} 224 \mathrm{cgc}$ & g4901c & $\begin{array}{c}\text { Pol-IN } \\
\text { (SA1D2prox) }\end{array}$ & $\mathrm{R} 224 \mathrm{cgc}$ \\
\hline \multirow[t]{4}{*}{ c4910t } & $\begin{array}{c}\text { Pol-IN } \\
\text { (SA1D2prox) }\end{array}$ & Y227tat & c4910t & $\begin{array}{c}\text { Pol-IN } \\
\text { (SA1D2prox) }\end{array}$ & Y227tat & & & \\
\hline & & & & & & c4916t & $\begin{array}{c}\text { Pol-IN } \\
\text { (SA1D2prox) }\end{array}$ & D229gat \\
\hline & & & & & & c5030t & $\begin{array}{c}\text { Pol-IN } \\
\text { (SA1D2prox) }\end{array}$ & I267att \\
\hline & & & & & & $\mathrm{t} 5132 \mathrm{a}$ & Vif & $\mathrm{I} 31 \mathrm{~N}$ \\
\hline
\end{tabular}


Table 3. Cont.

\begin{tabular}{|c|c|c|c|c|c|c|c|c|}
\hline \multicolumn{3}{|c|}{ NL-cgcad-1 } & \multicolumn{3}{|c|}{ NL-cgcad-2 } & \multicolumn{3}{|c|}{ NL-cgcad-4 } \\
\hline $\begin{array}{c}\mathrm{Nt} \\
\text { Change }\end{array}$ & Region & $\begin{array}{l}\text { NS/S Change } \\
\text { in the Region }\end{array}$ & $\begin{array}{l}\text { Nt } \\
\text { Change }\end{array}$ & Region & $\begin{array}{l}\text { NS/N Change } \\
\text { in the Region }\end{array}$ & $\begin{array}{c}\text { Nt } \\
\text { Change }\end{array}$ & Region & $\begin{array}{l}\text { NS/N Change } \\
\text { in the Region }\end{array}$ \\
\hline \multirow[t]{5}{*}{$\mathrm{t} 5223 \mathrm{c}$} & Vif & D61gac & t5223c & Vif & D61gac & & & \\
\hline & & & g5310a & Vif & R90aga & & & \\
\hline & & & g5593del & $* 1$ & & g5593del & 1 & \\
\hline & & & & *2 & & & 2 & \\
\hline & & & & & & $\mathrm{a} 5613 \mathrm{~g}$ & *2 & \\
\hline \multirow[t]{2}{*}{$\begin{array}{c}\text { 5632- } \\
\text { 5717del }\end{array}$} & *2 & & & & & & & \\
\hline & & & & & & g5865a & Tat & K12aaa \\
\hline c6633t & Env & $\mathrm{T} 138 \mathrm{I}$ & c6633t & Env & $\mathrm{T} 138 \mathrm{I}$ & & & \\
\hline t6643c & Env & N141aac & & & & & & \\
\hline c6652t & Env & S144agt & & & & & & \\
\hline c6830a & Env & P204T & c6830a & Env & P204T & & & \\
\hline \multirow[t]{2}{*}{ g6938a } & Env & V240I & g6938a & Env & V240I & g6938a & Env & V240I \\
\hline & & & c7531t & Env & I437att & & & \\
\hline
\end{tabular}

${ }^{*} 1$, the last seven of the amino acid sequence of Vif (SHTMNGH) was changed to AIQ. *2, a large deletion in Vpr. Bold letters show adaptive mutations that change the Vif expression levels of a parental NL-R224cgc clone. Nt, Nucleotide; NS, non-synonymous; S, synonymous.

\subsection{Characteristics of Viral Clones Obtained from Excessive-Vif Type NL-P238ccg Adaptation}

We examined the replication potential of adapted clones (NL-ccgad) obtained through an adaptation experiment of NL-P238ccg (Figure 1C). As shown in Figure 5A, NL-P238ccg grew poorly compared to NL4-3, as we described previously [38]. The growth kinetics of NL-ccgad clones 5, 8, 10, and 13 were almost the same as that of NL4-3. While an enhanced growth potential of NL-ccgad-6 was observed compared to that of a parental NL-P238ccg, NL-ccgad clones 1 and 7 exhibited a reduced replication ability (Figure 5A). The Vif expression levels for most of the NL-ccgad clones tested were similar to that of WT NL4-3, whereas NL-ccgad clones 1 and 6 showed a considerably reduced level of Vif expression (Figure 5B). We carried out sequence analyses of NL-ccgad clones 5, 8, and 10 which grow as well as NL4-3. These three clones are revertants that have P238cca instead of P238ccg, and also carry several synonymous and non-synonymous mutations within the Vif, Vpr, and Env regions (data not shown). As shown in Table 4, low-Vif type NL-ccgad clones 1 and 6 carry mutations, G237ggg and D229gat, respectively, within the SA1D2prox region. As mentioned above, the Vif expression level was decreased by the D229gat mutation (Figure 3B). The difference in growth potential between NL-ccgad clones 1 and 6 may be explained by the presence of mutation(s) that can affect the Vif expression level and/or viral replication ability. 


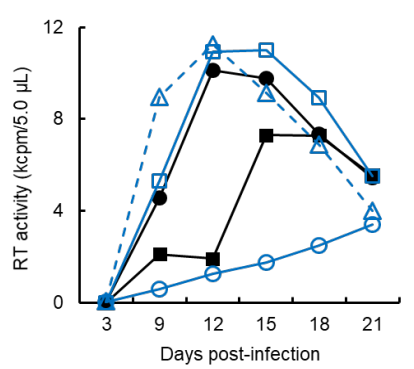

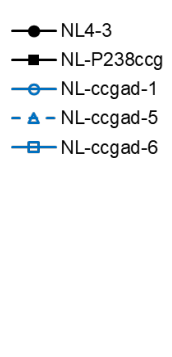
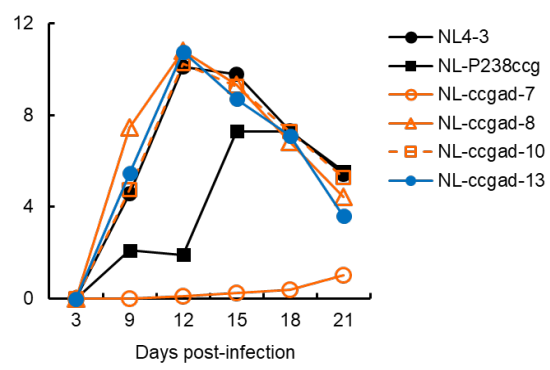

B

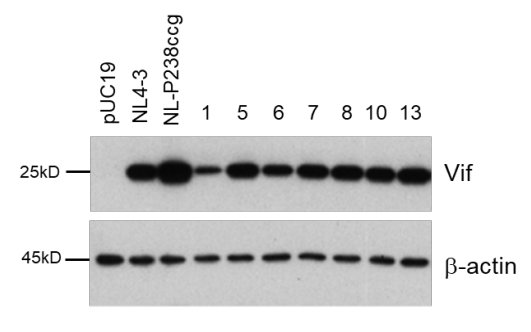

Figure 5. Characteristics of viral clones constructed from adapted NL-P238ccg. (A) Growth kinetics. Viruses prepared from HEK 293T cells transfected with the indicated pro-viral clones were inoculated into H9 cells $\left(1 \times 10^{4}\right.$ RT units $/ 10^{5}$ cells $)$. Virus replication was monitored by the virion-associated RT activity in the culture supernatants. This experiment was performed once to select viral clones that have the ability to grow in cells. Viral replication kinetics of WT NL4-3 and a parental NL-P238ccg are presented in both panels for easy comparison. (B) Vif expression levels. Western blotting analysis was carried out as described in Figure 3B. Numbers correspond to those for the adapted clones shown in panel (A). Representative data from two independent experiments are shown.

Table 4. Mutations found in adapted clones with decreased Vif expression levels compared to that in the NL-P238ccg clone.

\begin{tabular}{|c|c|c|c|c|c|}
\hline \multicolumn{3}{|c|}{ NL-ccgad-1 } & \multicolumn{3}{|c|}{ NL-ccgad-6 } \\
\hline Nt Change & Region & NS/S Change in the Region & Nt Change & Region & NS/N Change in the Region \\
\hline g2923a & Pol-RT & R125K & & & \\
\hline \multirow[t]{3}{*}{ g4071a } & Pol-RT & A508T & & & \\
\hline & & & g4566a & Pol-IN & V313I \\
\hline & & & c4916t & $\begin{array}{c}\text { Pol-IN } \\
\text { (SA1D2prox) }\end{array}$ & D229gat \\
\hline $\mathrm{a} 4940 \mathrm{~g}$ & $\begin{array}{c}\text { Pol-IN } \\
\text { (SA1D2prox) }\end{array}$ & G237ggg & & & \\
\hline a4943g & $\begin{array}{c}\text { Pol-IN } \\
\text { (SA1D2prox) }\end{array}$ & P238ccg & a4943g & $\begin{array}{c}\text { Pol-IN } \\
\text { (SA1D2prox) }\end{array}$ & P238ccg \\
\hline g5090a & $\begin{array}{l}\text { Pol-IN } \\
\text { Vif }\end{array}$ & $\begin{array}{l}\text { E287gaa } \\
\text { R17K }\end{array}$ & & & \\
\hline a5242g & Vif & T68A & a5242g & Vif & $\mathrm{T} 68 \mathrm{~A}$ \\
\hline \multirow[t]{4}{*}{ a5315g } & Vif & K92R & & & \\
\hline & & & $\mathrm{t} 5379 \mathrm{c}$ & Vif & D113gac \\
\hline & & & $\mathrm{a} 5602 \mathrm{~g}$ & Vif & $\mathrm{T} 188 \mathrm{~A}$ \\
\hline & & & & Vpr & Y15C \\
\hline 5627-5651del & *1 & & & & \\
\hline g5797a & *1 & & & & \\
\hline a5820g & *1 & & a5820g & Vpr & R88G \\
\hline g6625a & Env & K135aag & g6625a & Env & K135aag \\
\hline \multirow[t]{2}{*}{ g7032a } & Env & $\mathrm{R} 271 \mathrm{~K}$ & g7032a & Env & $\mathrm{R} 271 \mathrm{~K}$ \\
\hline & & & a7180c & Env & K320N \\
\hline
\end{tabular}

${ }^{*} 1$, a large deletion in Vpr. Bold letters show adaptive mutations that change the Vif expression levels of a parental NL-P238ccg clone. Nt, Nucleotide; NS, non-synonymous; S, synonymous.

\subsection{Identification of Adaptive Mutations Associated with the Variation in Vif Expression Level}

In total, all adapted clones with the altered Vif expression level carry a mutation or mutations within the SA1D2prox region as follows: NL-gatad clones 1, 6, and 7 have the R269Kaag mutation; NL-cgcad clones 1, 2, and 4 carry either the Y227tat mutation or the D229gat/I267att double mutation; NL-ccgad clones 1 and 6 possess the G237ggg mutation and the D229gat mutation, respectively (Tables 2-4). To determine whether these mutations affect the Vif expression level, we newly constructed pro-viral clones carrying two mutations, a parental mutation and a mutation found in the SA1D2prox region. The Vif expression levels were analyzed using HEK 293T cells transfected with pro-viral clones 
(Figure 6). A pro-viral clone (NL-gat+aag), bearing D229gat (parental) and R269Kaag (adaptive) mutations, exhibited an increase in the Vif expression level compared to NLD229gat, but to a lesser extent than NL4-3. The introduction of either Y227tat or D229gat into a parental NL-R224cgc clone (NL-cgc+tat and NL-cgc+gat) markedly reduced the Vif expression level relative to NL-R224cgc and NL4-3. Similarly, the Vif expression levels were remarkably decreased for clones NL-ccg+ggg and NL-ccg+gat carrying a parental P238ccg mutation and either G237ggg or D229gat (Figure 6). While we have already shown the effect of mutations R269Kaag and D229gat on the Vif expression level [38], we newly identified mutations Y227tat and G237ggg that strongly reduce the Vif expression level in this study. In this regard, we have previously shown that the vif mRNA expression level of the Y227Fttc mutant clone is similar to that of WT NL4-3 [38]. The single nucleotide change of WT Y227tac to either Y227tat or Y227Fttc differently affects the Vif expression level. This result implies the importance of nucleotide sequence in the SA1D2prox region, although whether the Y227tat mutation alone can alter the Vif expression level needs to be examined. In sum, the change in the Vif expression levels of five newly constructed pro-viral clones (Figure 6) were well correlated with the alteration in those of adapted viral clones (Figures 3-5). These results indicate that mutations found within the SA1D2prox region are indeed adaptive mutations, suggesting a strong association between the nucleotide sequence of the SA1D2prox region and the determination of the Vif expression level.
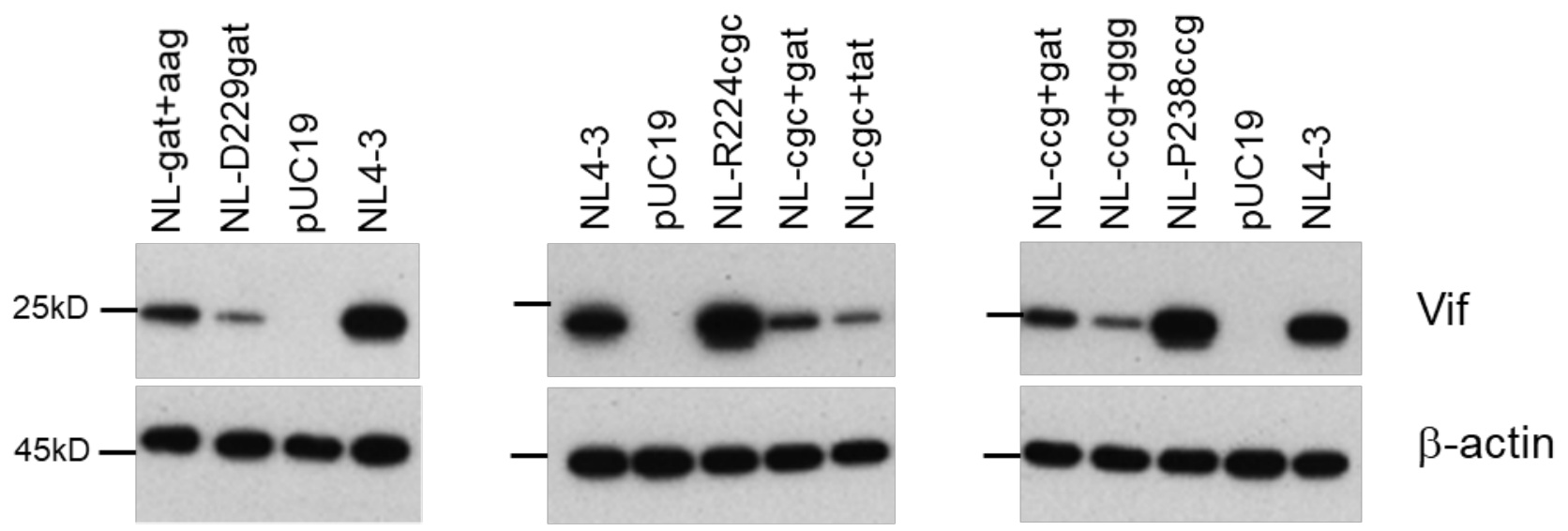

Figure 6. Effects of adaptive mutations within SA1D2prox on the Vif expression level. HEK 293T cells were transfected with the indicated pro-viral clones. On day 1 post-transfection, cell lysates were prepared for Western blotting analysis using anti-Vif and anti- $\beta$-actin antibodies. Adaptive mutations that change the Vif expression levels found within the SA1D2prox region of NL-D229gat, NL-R224cgc, and NL-P238ccg are shown in Tables 2-4, respectively. Representative data from two independent experiments are shown. gat, D229gat; aag, R269Kaag; cgc, R224cgc; tat, Y227tat; ccg, P238ccg; ggg, G237ggg.

\subsection{Effect of Adaptive Mutations within the SA1D2prox Region on the Splicing Pattern}

We have previously demonstrated that nSNMs within the SA1D2prox region do not affect the total HIV-1 mRNAs production levels but change the overall splicing pattern, which in turn alters the vif mRNA/Vif protein expression level and viral growth potential [37-39]. Moreover, we and others noticed the inverse correlation between vif mRNA/protein and opr mRNA/protein expression levels [37,38,54]. To facilitate evaluation of the changes in the splicing pattern, we newly constructed a minigenome (pcNLmini-RI) possessing SA1 and SA2 that are important for vif and vpr mRNA production, respectively (Figure 7A). The minigenome contains the SD1 site, the SA1D2prox region, the entire vif sequence, and the partial vpr sequence from the Vpr start codon to the authentic EcoRI site of NL4-3. Combinatorial mutation sets of parental mutations (D229gat, R224cgc, and P238ccg) and adaptive mutations (R269Kaag, Y227tat, D229gat, and G237ggg) identical to those in the pro-viral clones described above were introduced into the minigenome to analyze the splicing pattern. We first ascertained whether the minigenomes constructed display the altered Vif expression levels as observed for pro-viral clones (Figure 6). The Vif expression 
level for minigenomes was determined using transfected HEK 293T cells (Figure 7B). In agreement with the results obtained using pro-viral clones (Figure 6), the Vif expression levels varied among minigenomes carrying the mutations (Figure 7B). A minigenome carrying both D229gat and R269Kaag exhibited an increased level of Vif expression relative to that with D229gat alone. The reduction in Vif expression levels was evident for minigenomes containing an adaptive mutation of Y227tat, D229gat, or G237ggg relative to the parental R224cgc and P238ccg constructs. The alteration in the Vif expression level for the minigenomes tested correlated very well with that of pro-viral clones (Figures 6 and 7).

\section{A pcNLmini-RI}

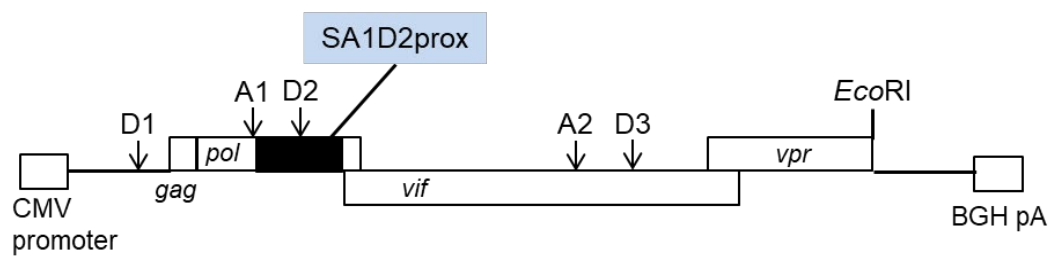

B

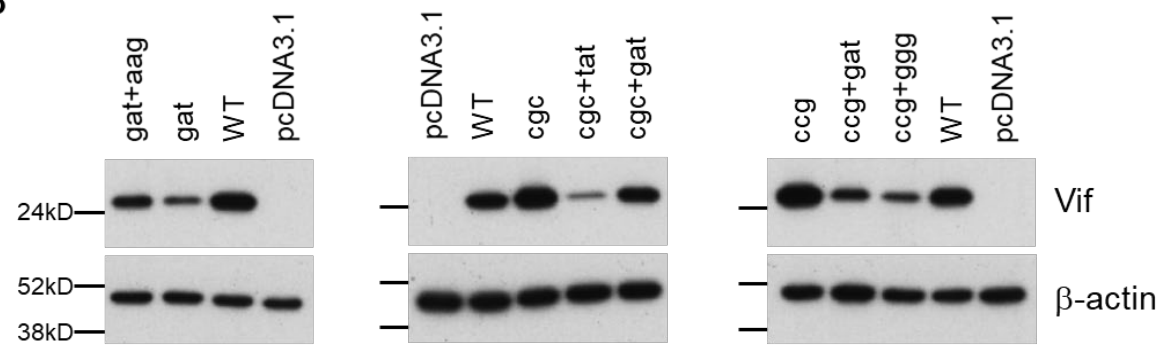

Figure 7. Vif expression levels of a newly constructed minigenome (pcNLmini-RI) vector. (A) Organization of the minigenome. The pcNLmini-RI vector was constructed as described in the Materials and Methods. Splicing (D1, A1, D2, A2, D3) and EcoRI sites in the minigenome vector corresponding to those in the authentic HIV-1 NL4-3 clone are shown. A black box indicates the SA1D2prox region. CMV promoter, cytomegalovirus enhancer-promoter; BGH pA, bovine growth hormone polyadenylation sequence. (B) Vif expression levels of the minigenome vectors carrying various mutations. HEK $293 \mathrm{~T}$ cells were transfected with the indicated minigenome vectors, and on day 1 post-transfection, cell lysates were prepared for Western blotting analysis using anti-Vif and anti- $\beta$-actin antibodies. Mutations introduced into the minigenome vector were the same as those shown in Figure 5. An empty vector (pcDNA3.1) was used as a negative control. Representative data from two independent experiments are shown. WT, wild type; gat, D229gat; aag, R269Kaag; cgc, R224cgc; tat, Y227tat; ccg, P238ccg; ggg, G237ggg.

Next, we determined the change in splicing patterns by mutations using the minigenome constructs. HEK 293T cells were transfected with various minigenomes, cells were lysed for RNA extraction, and then cDNA samples were synthesized using oligo(dT) primer. Semiquantitative PCR was carried out to amplify distinct $200-400$ bp splicing products (full-length, D1/A1, D1/A1-D2/A2, and D1/A2) using the specific primer sets depicted in Figure 8A. D1 (Figure 8A) and the gapdh products were also amplified simultaneously, and analyzed as controls for transfection efficiency and total RNA amount, respectively. As shown in Figure 8B, the low-Vif type D229gat displayed a decrease in the D1/A1 splicing product and an undetectable level of D1/A1-D2/A2 product relative to WT without any mutations, whereas the expression levels of full-length and D1/A2 products in D229gat were increased (Figure 8B). As expected, the D1/A1 product was increased in a minigenome with both D229gat and R269Kaag (gat+aag) compared to a parental minigenome D229gat. While the D1/A1-D2/A2 product for the gat+aag construct was still undetectable, a decrease in full-length and D1/A2 products was observed relative to D229gat (Figure 8B). 
For excessive-Vif type R224cgc and P238ccg, the changes in the splicing pattern by adaptive mutations were also observed depending on the alteration in the Vif expression level (Figure 8C). The D1/A1 and D1/A1-D2/A2 products by R224cgc and P238ccg were elevated compared to that of WT, whereas a decrease in full-length and D1/A2 products was observed for both of them. Minigenomes carrying an adaptive mutation along with a parental mutation (R224cgc+Y227tat, R224cgc+D229gat, P238ccg+D229gat, and P238ccg+G237ggg) exhibited decreased levels of D1/A1 and D1/A1-D2/A2 products but increased levels of full-length and D1/A2 products relative to each parental minigenome (Figure $8 \mathrm{C}$ ). These results, in good agreement with previous reports, showed that while the change in D1/A1 production level by mutations in the SA1D2prox correlates with the alteration in the Vif expression level, the D1/A2 production level is inversely correlated with the D1/A1 production level. Taken together, these results suggest that adaptive mutations identified within the SA1D2prox region which alter the Vif expression level affect the mRNA production levels mainly via effects on the splicing.

A

pcNLmini-R

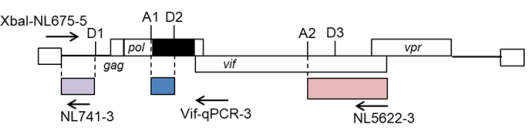

Primer pair (Xbal-NL675-5 and Vif-qPCR-3)

$\square_{132 \mathrm{bp}}^{\text {73bp }} \stackrel{\text { 50bp }}{\square_{110 \mathrm{bp}}} 365 \mathrm{bp}$ (full)

73bp

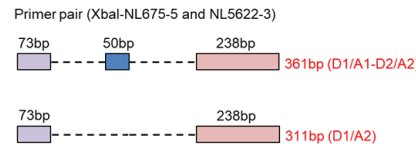

C
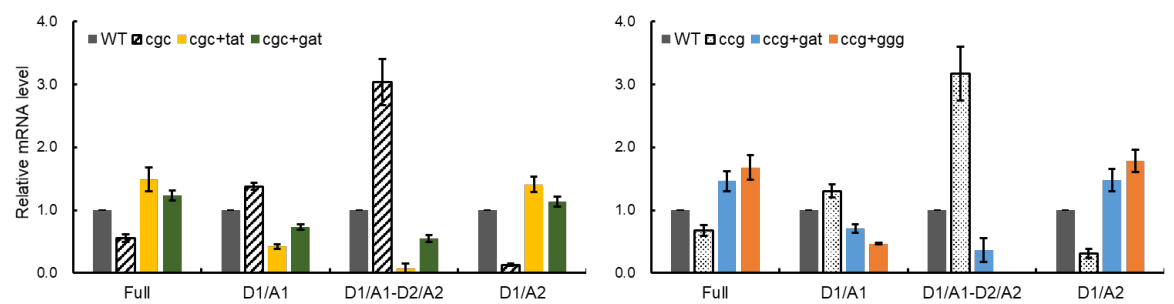

Figure 8. Effects of adaptive mutations on mRNA production. (A) mRNA produced from the minigenome. Splicing sites on the minigenome are presented. The primers used for the splicing pattern analysis are indicated by arrows with their names (upper part). Colored boxes show exons produced by splicing at various sites. In the lower part, splicing products analyzed using the primer sets are presented along with their lengths. Broken and solid lines show regions with and without splicing, respectively. (B,C) Changes in the splicing pattern of parental clones (gat, cgc, and ccg) by their adaptive mutations. Total RNA was prepared from HEK 293T cells transfected with the indicated minigenome vectors and subjected to semiquantitative RT-PCR using the primer pairs shown in (A). The signal intensities of semiquantitative RT-PCR products were quantitated from three independent experiments. The intensities of the indicated mRNAs in each sample were normalized to those of all viral mRNAs (D1) and gapdh mRNA. The normalized mRNA intensities in each sample relative to those of WT are presented.

\subsection{Chronological Changes in the Vif Expression Levels by Mutations within SA1D2prox in Infected Individuals}

We have previously found various nSNMs that can alter the Vif expression level by examining the SA1D2prox sequence obtained from the HIV-1 sequence database (Los Alamos National Laboratory) [37-39]. It was of interest, in the present study, to determine whether changes in the nucleotide sequence of SA1D2prox can be observed for samples obtained chronologically from patients and whether the changes influence the Vif expression level. For this purpose, we used the next-generation sequence data from plasma samples collected from protease inhibitor (PI)-resistant and integrase inhibitor (RAL)-resistant patients 
at different time points [55]. Pro-viral clones designated NL-pC2 and NL-pC3, which carry two and three nucleotide variations relative to NL4-3, respectively, represent a consensus sequence of SA1D2prox in HIV-1 subtype B strain (Figure 9A) [39]. The vif transcript levels for NL-pC2 and NL-pC3 were similar and slightly lower than that for NL4-3 (around 0.7 for NL-pC3 relative to that of NL4-3) [39]. The sequence data of SA1D2prox were obtained at 10 and 6 different time points for PI-resistant patients PI1and PI4, respectively (Figure 9B,C), and at 7 and 3 different time points for RAL-resistant patients RAL4 and RAL5, respectively (Figure 9D,E). Changes in the SA1D2prox sequence were observed over the survey period for the PI- and RAL-resistant patient samples used. Pro-viral clones that have the nucleotide sequence of SA1D2prox obtained were constructed using NL4-3 as a backbone, and were divided into groups (A-C or D) based on the sequence identity, e.g., PI1-A and PI1-B (Figure 9B-D). These pro-viral clones were transfected into HEK 293T cells, and cell lysates prepared were subjected to Western blotting analyses. As shown in Figure 10, although no significant alteration was observed for the Vif expression levels among PI1-A to PI1-C and PI4-A to PI4-D samples, these clones exhibited a moderately decreased level of Vif expression compared to their reference clones, NL-pC2 and NL-pC3. Importantly, these decreases in the Vif expression levels were expected, because we have already shown that many variations, if not all, found in the sequences for PI1 and PI4 samples lower the vif transcript/mRNA levels relative to NL4-3 [37,38]: D232gac (0.47), L242ctt (0.28), Q252cag (0.69), and K258aag (0.93) for PI1; R228aga (0.28), D253gac (1.03), R263agg (0.60), and K266aaa (0.75) for PI4. Interestingly, the Vif expression levels were different among the RAL4-A to RAL4-D and RAL5-A to RAL5-C samples, and varied during the survey period (Figure 10). For the RAL4 samples, the Vif expression level of RAL4-C was increased relative to that of RAL4-B. It is reasonably assumed that this may be due to the presence of V249gtg within the RAL4-C sequence which we have shown to increase the vif transcript level compared to NL4-3 (1.83 on average) [39]. These results showed that the SA1D2prox sequence can be chronologically changed and affect the Vif expression level during in vivo replication.

A

NL4-3

$\mathrm{NL}-\mathrm{pC} 2$

$\mathrm{NL}-\mathrm{pC} 3$

NL4-3

$\mathrm{NL}-\mathrm{pC} 2$

$\mathrm{NL}-\mathrm{pC} 3$

NL4-3

$\mathrm{NL}-\mathrm{pC} 2$

$\mathrm{NL}-\mathrm{pC} 3$

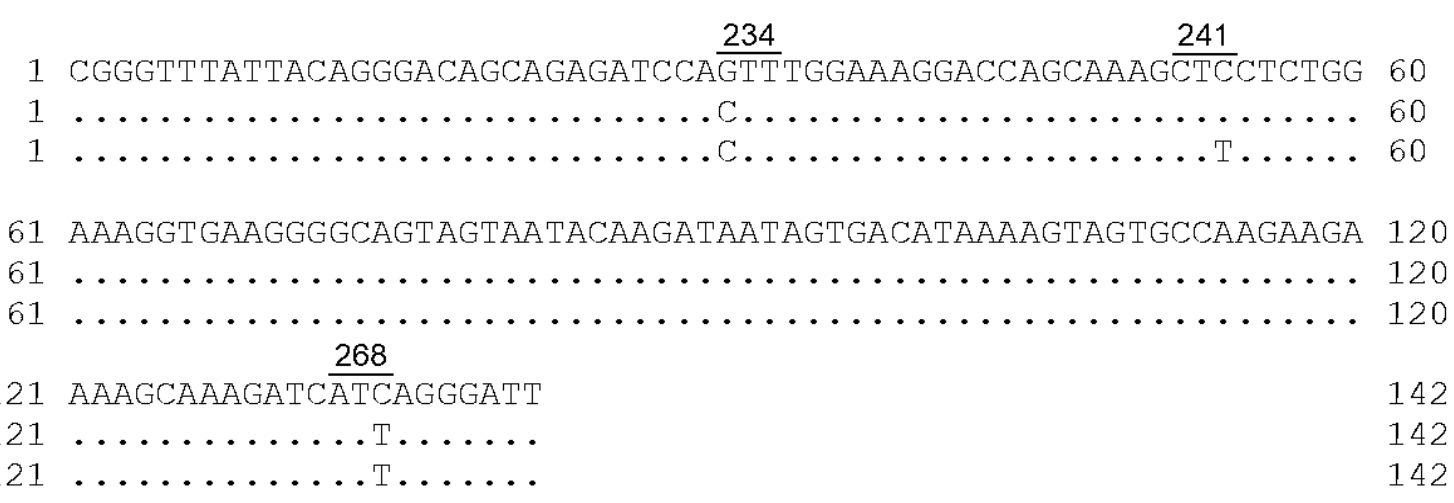

Figure 9. Cont. 
B

Clone name

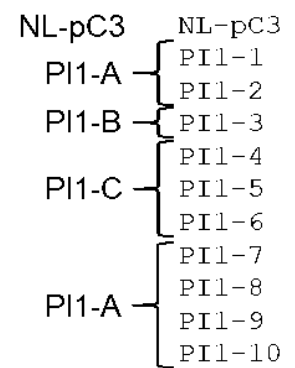

NL-pC3

PII-1

PIl-2

PI $1-3$

PI $1-4$

PII-5

PI $1-6$

PII-7

PII-8

PII-9

PII-10

NL-pC3

PIl-1

PII-2

PIl-3

PII-4

PII-5

PI $1-6$

PII-7

PI $1-8$

PI $1-9$

PIl-10

$\begin{array}{ll}1 & C G \\ 1 & \ldots \\ 1 & \ldots \\ 1 & \ldots \\ 1 & . \\ 1 & . \\ 1 & . \\ 1 & . \\ 1 & . \\ 1 & . \\ 1 & .\end{array}$

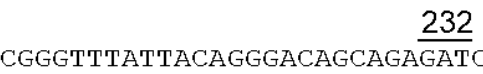

232

242

(1)

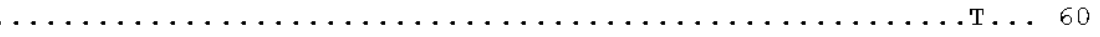

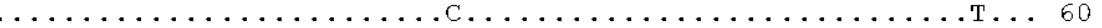

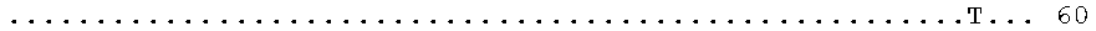

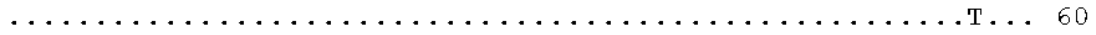

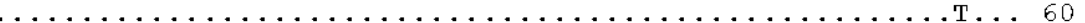

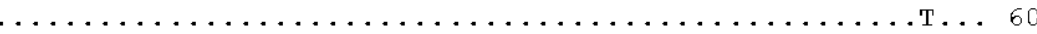

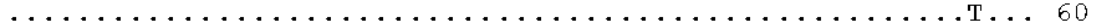

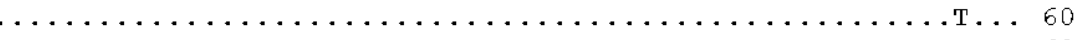

252

258

61 AAAGgTGAAGGGGCAGTAGTAATACAAGATAATAGTGACATAAAAGTAGTGCCAAGAAGA 120

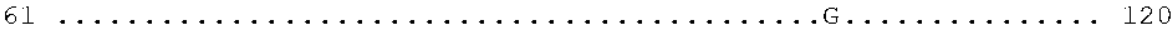

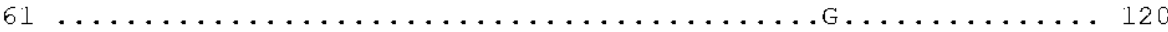

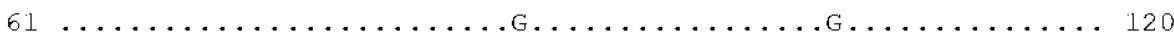

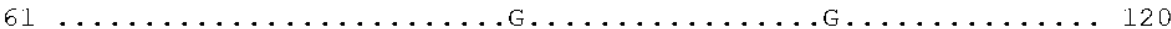

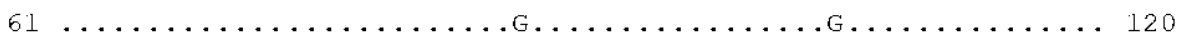

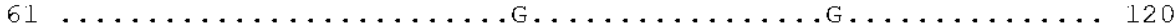

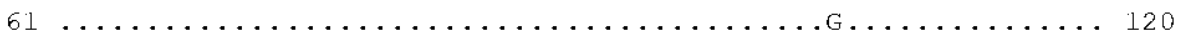

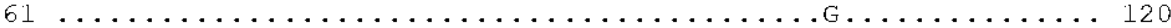

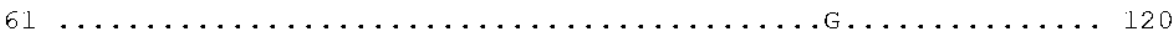

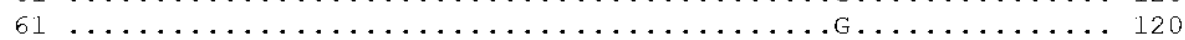

121 AAAGCAAAGATCATTAGGGATT 142

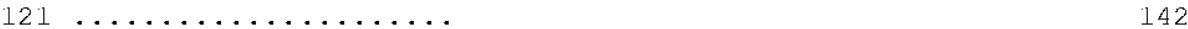

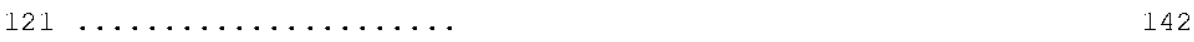

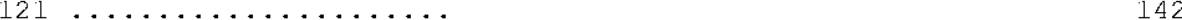

$121 \ldots \ldots \ldots \ldots \ldots \ldots \ldots \ldots \ldots \ldots \ldots$

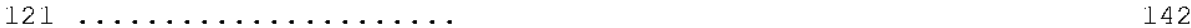

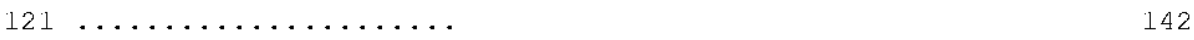

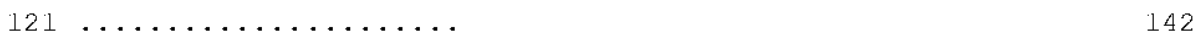

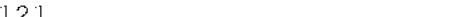

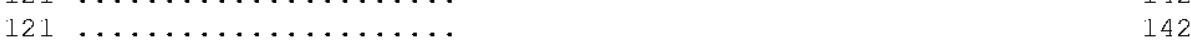

$121 \ldots \ldots \ldots \ldots \ldots \ldots \ldots \ldots \ldots \ldots \ldots$

C

Clone name

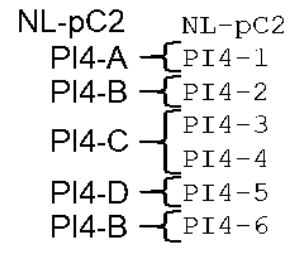

$\mathrm{NL}-\mathrm{pC} 2$

PI 4-1

PI 4-2

PI 4-3

PI 4-4

PI 4-5

PI 4-6

$\mathrm{NL}-\mathrm{pC} 2$

PI 4-1

PI 4-2

PI 4-3

PI 4-4

PI 4-5

PI 4-6

\section{8}

1 CGGGTTTATTACAGGGACAGCAGAGATCCACTTTGGAAAGGACCAGCAAAGCTCCTCTGG 60

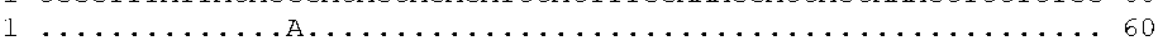

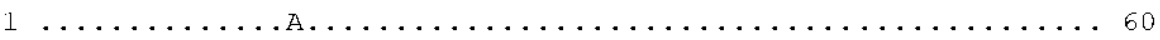

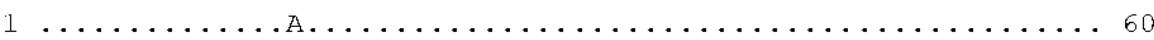

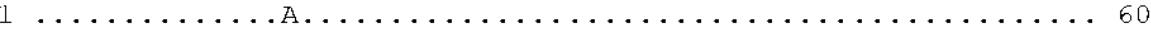

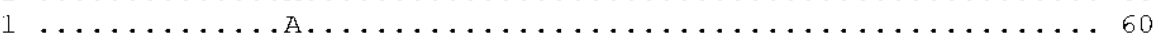

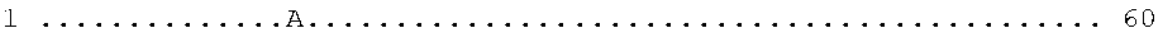

253

263

61 AAAgGTGAAGGGGCAGTAGTAATACAAGATAATAGTGACATAAAAGTAGTGCCAAGAAGA 120

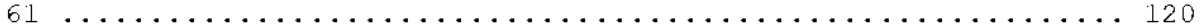

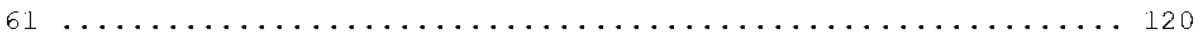

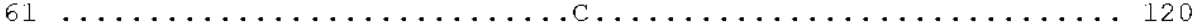

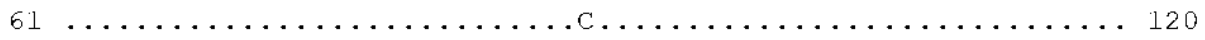

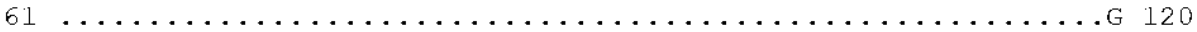

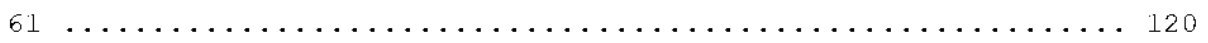

\section{6}

121 AAAGCAAAGATCATTAGGGATT 142

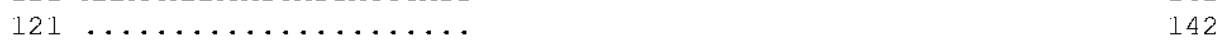

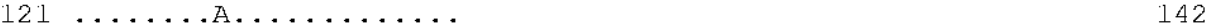

$121 \ldots \ldots \ldots \ldots \ldots \ldots 142$

$121 \ldots \ldots \ldots \ldots \ldots \ldots \ldots \ldots$

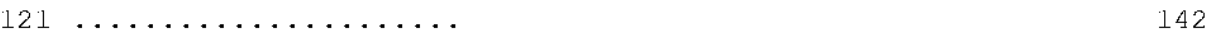

$121 \ldots \ldots \ldots \ldots \ldots \ldots 142$

Figure 9. Cont. 
$\mathrm{D}$

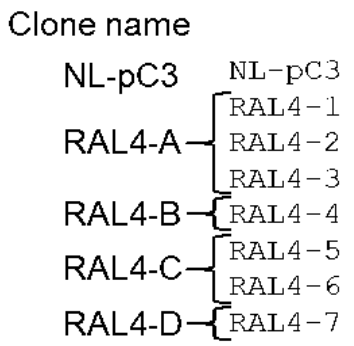

$\mathrm{NL}-\mathrm{pC} 3$

RAL4-1

RAL 4-2

RAL 4-3

RAL 4-4

RAL4-5

RAL4-6

RAL4-7

$\mathrm{NL}-\mathrm{pC} 3$

RAL4-1

RAL 4-2

RAL 4-3

RAL4-4

RAL4-5

RAL4-6

RAL 4-7
228

$232 \quad 234$

1 CGGGTTTATTACAGGGACAGCAGAGATCCACTTTGGAAAGGACCAGCAAAGCTTCTCTGG 60

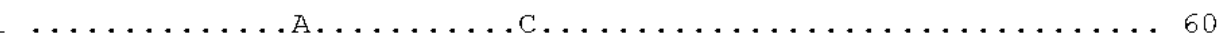

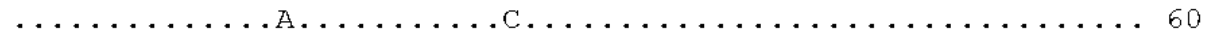

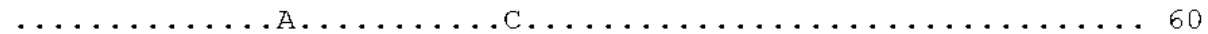

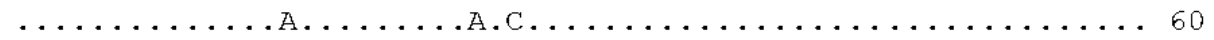

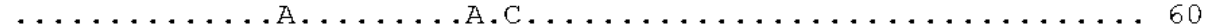

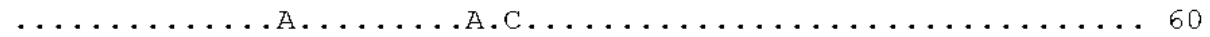

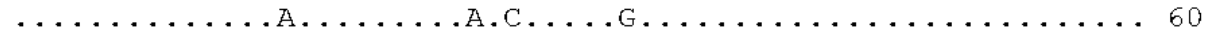

\section{$\underline{249} \quad 254 \quad 256 \quad 259$}

61 AAAGGTGAAGGGGCAGTAGTAATACAAGATAATAGTGACATAAAAGTAGTGCCAAGAAGA 120

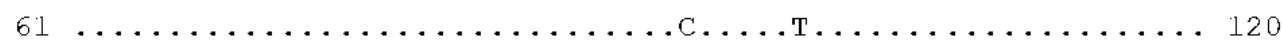

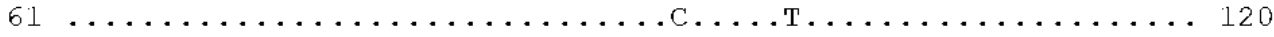

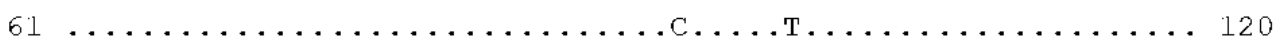

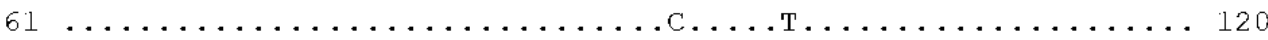

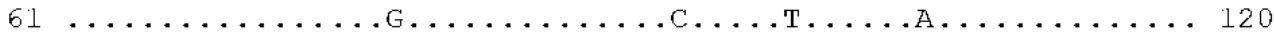

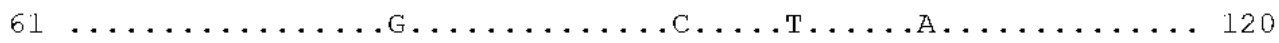

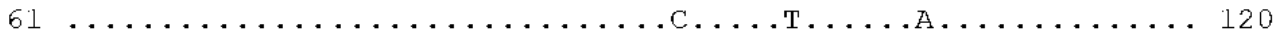

269

121 AAAGCAAAGATCATTAGGGATT

$121 \ldots \ldots \ldots \ldots \ldots \ldots \ldots 142$

$121 \ldots \ldots \ldots \ldots \ldots \ldots \ldots \ldots 14 \ldots \ldots \ldots$

$121 \ldots \ldots \ldots \ldots \ldots \ldots \ldots 142$

$121 \ldots \ldots \ldots \ldots \ldots \ldots \ldots 142$

$121 \ldots \ldots \ldots \ldots \ldots \ldots \ldots 142$

$121 \ldots \ldots \ldots \ldots \ldots \ldots 142$

$121 \ldots \ldots \ldots \ldots \ldots \ldots \ldots 142$

E

Clone name

NL-pC3

NL-pC3

NL-PC3

RAL5-B $\{$ RAL 5-2

RAL5-C $\{$ RAL $5-3$

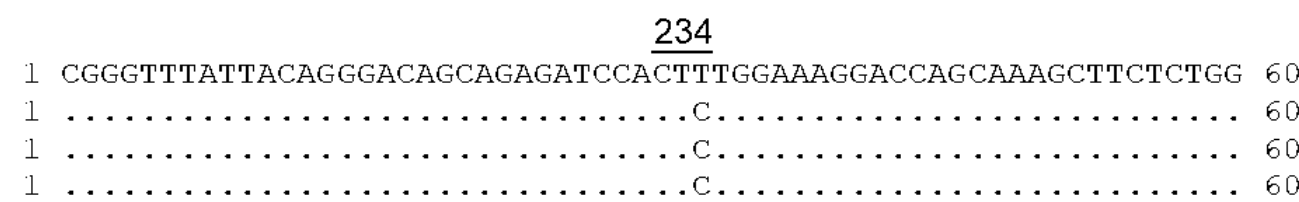

NL-pC 3
RAL5 -
RAL5 - 2
RAL5 - 3

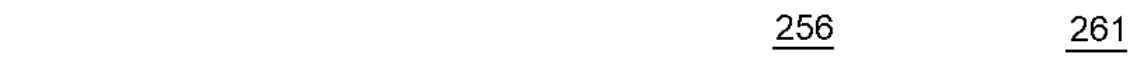

61 AAAgGTGAAGgGgCAgTAGTAATACAAGATAATAGTGACATAAAAGTAGTGCCAAGAAGA 120

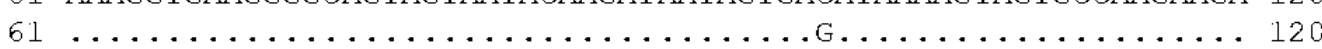

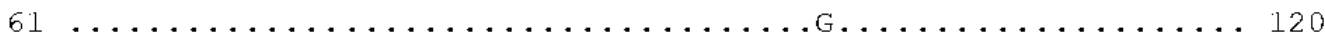

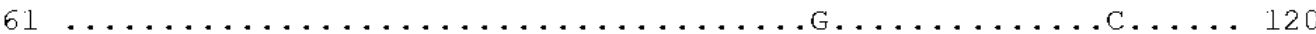

\begin{tabular}{|c|c|c|}
\hline & 269 & \\
\hline $\mathrm{NL}-\mathrm{pC} 3$ & 121 AAAGCAAAGATCATTAGGGATT & 142 \\
\hline RAL5-1 & $121 \quad \ldots \ldots \ldots \ldots \ldots$ AA. & 142 \\
\hline RAL5-2 & $121 \quad \ldots \ldots G \ldots \ldots \ldots$ AA. . & 142 \\
\hline RAL5-3 & $121 \ldots \ldots \ldots \ldots A \ldots \ldots$ & 142 \\
\hline
\end{tabular}

Figure 9. SA1D2prox sequences of NL4-3, its derivatives, and clinical samples. Sequence alignments were carried out using Genetyx Ver. 15. Numbers and underlines above the sequences represent Pol-IN amino acid positions (NL4-3 numbering) and codons, respectively. (A) Sequences of NL4-3, NL-pC2, and NL-pC3. NL-pC2 and NL-pC3 clones have 2 and 3 nucleotide substitutions, respectively, which are major nucleotides at each position in the SA1D2prox sequence within the HIV-1 subtype B population. (B,C) Chronological changes of the SA1D2prox sequence in samples obtained at different time points from PI-resistant patients PI1 (B) and PI4 (C). Sequence data were published previously [55] (DDBJ; PRJDB3502). Clone name was designated PI1-A, -B, and -C (B) and PI4-A, -B, -C, and -D (C) based on the sequence identity. (D,E) Chronological changes of the SA1D2prox sequence in samples obtained at different time points from RAL-resistant patients RAL4 (D) and RAL5 (E). Sequence data were published previously [55] (DDBJ; PRJDB3502). Clone name was designated RAL4-A, $-B$, and $-C(D)$ and RAL5-A, $-B$, and $-C$ (E) based on the sequence identity and the chronological order. 


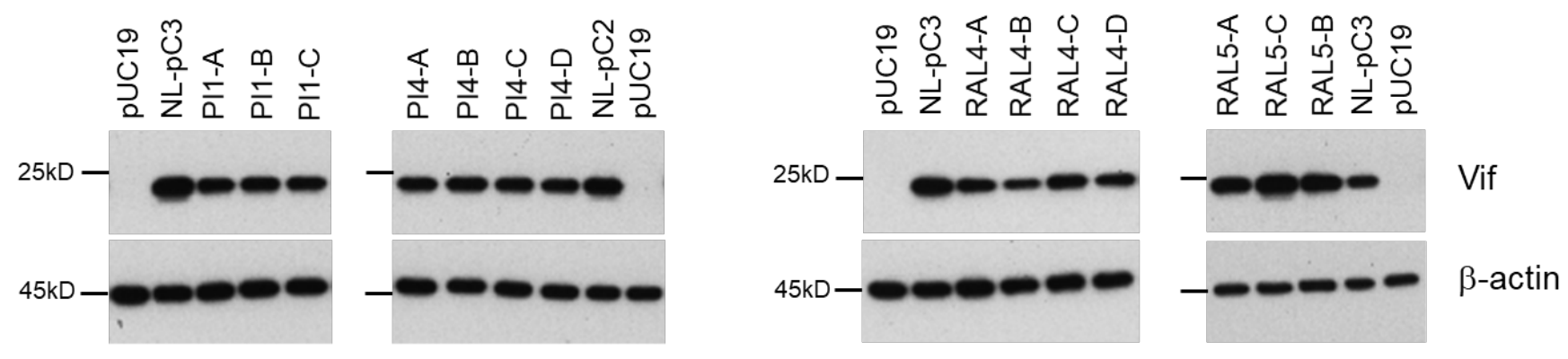

Figure 10. Vif expression levels of pro-viral clones carrying different SA1D2prox sequences in the HIV-1 NL4-3 genome. HEK 293T cells were transfected with the indicated pro-viral clones, and on day 1 post-transfection, cell lysates were prepared and subjected to Western blotting analysis using anti-Vif and anti- $\beta$-actin antibodies. Representative data from two independent experiments are shown.

\section{Discussion}

We have previously reported that the low-Vif type NL-D229gat and excessive Vif-type NL-R224cgc/P238ccg clones exhibit a reduced growth potential relative to WT NL4-3 depending on their Vif expression levels and cellular A3G expression level (Figure 2) [37-39]. In this study, we sought to determine how these clones adapt under the growth restrictive condition imposed by $\mathrm{H} 9$ cells with a high expression level of A3G, especially focusing on the fluctuation in the Vif expression level. Some of the adapted clones constructed exhibited altered Vif expression levels relative to the parental clone (Figures 3-5) and carry mutations within SA1D2prox (Tables 1-4). Indeed, these mutations found in the region were responsible for the alteration in the Vif expression level (Figure 6). Minigenomes newly constructed recapitulated changes in the Vif expression level by parental and adaptive mutations observed for pro-viral clones. Semiquantitative PCR analyses were carried out using minigenomes to examine the effect of mutations on the splicing patterns. In agreement with previous reports $[38,39,54]$, the alteration in the Vif expression level correlated well with the change in the D1/A1 splicing production levels, and there was an inverse correlation between the D1/A1 and D1/A2 production levels (Figures 7 and 8). Moreover, we analyzed the Vif expression level using NL-based pro-viral clones that carry the SA1D2prox sequence obtained from plasma samples collected from patients at different time points. The results showed that, even in intra-patient samples, the SA1D2prox sequence can fluctuate chronologically and variations in the sequence can affect the Vif expression levels (Figures 9 and 10). Taken together, the SA1D2prox nucleotide sequence is associated with viral adaptation through tuning the Vif expression level vital for the HIV-1 replication property.

We have previously proposed that there may be an appropriate Vif expression level required for efficient HIV-1 replication, because a viral clone with a low level of Vif expression fails to sufficiently degrade A3G, and because viral infectivity and virion production are inhibited by a high level of Vif expression $[19,38,49,50]$. Even though moderate alterations in vif production level by nSNMs within SA1D2prox (around 0.2 around 1.8 relative to WT NL4-3) do not significantly affect HIV-1 replication, low-Vif type NL-D229gat and excessive-Vif type NL-R224cgc/NL-P238ccg, which exhibit drastic changes in Vif level below 0.1 and over 2.5 for vif production relative to WT NL4-3, respectively, have the impaired replication ability as reported [38]. Adapted clones were generated from viruses that emerged in long-term cultures of $\mathrm{H} 9$ cells infected with low- and excessive-Vif type clones. Importantly, all replication-competent adapted viruses with altered Vif expression levels invariably harbored single-nucleotide changes within SA1D2prox. This implies that, during adaptation, HIV-1 quickly repairs an inappropriate range of Vif expression level, which resulted in an inferior viral replication, through acquiring single-nucleotide mutations, mostly synonymous mutations, within SA1D2prox. It has been reported that the introduction of numerous synonymous mutations throughout the HIV-1 genome, especially into the central region of the genome, results in replication defects via splicing 
perturbation [56]. Several lines of evidence showed that synonymous mutations can affect pivotal biological processes such as RNA splicing, RNA stability, and translation efficiency [56-58], for review, see [59,60]. This implies that synonymous mutations would be biologically relevant and exposed to selective forces. Thus, synonymous mutations can contribute to HIV-1 adaptation and evolution [60]. The results in this study provide a good example of this phenomenon. The SA1D2prox sequence contains splicing sites SA1/SD2 and is located within the Pol-integrase region, and thus amino acid changes by substitutions in the SA1D2prox sequence can affect the integrase function. For HIV-1 adaptation, it is reasonable to repair replication defects involved in Vif expression levels through synonymous mutations within the SA1D2prox region without adverse effects on the integrase function.

The difference in growth potential was observed among adapted clones that exhibit similar Vif expression levels (Figures 3-5). We found various mutations throughout the genomes of these adapted clones (Tables 1-4). It is obvious that, since Vif expression level is not a sole determinant for HIV-1 replication ability in cells, some of these mutations can positively or negatively affect the viral replication ability independent of the Vif expression level. The effect of such mutations on the viral replication ability is currently under investigation in our laboratory. Another intriguing project is to investigate the effect of variation in cellular A3G expression level on an adaptation of HIV-1 clones with different Vif expression levels. This is ongoing research in our laboratory, in which we have been working on adaptation experiments using our SA1D2prox mutant clones and CEM-SS cells expressing a low level of A3G.

Chronological alterations in Vif expression level by changes of SA1D2prox nucleotide sequence were confirmed also by using sequences of the region from patients' samples. This result may imply that the SA1D2prox nucleotide sequence continues to change to retain an appropriate Vif level in individuals. It would be intriguing to study whether the increase and decrease in the Vif expression level by changing the SA1D2prox nucleotide sequence is associated with viral adaptation and replication ability in individuals. However, it may be difficult to investigate the involvement of the Vif expression level in viral replication ability / adaptation in vivo, considering that correlations between the expression level of A3G or A3F in individuals and the viral replication level/the disease progression state remain quite controversial [5,61]. In addition, changes in D1/A1 splicing can affect D1/A2 splicing, suggesting that the Vif expression level can be influenced by the nucleotide sequence of region(s) distinct from SA1D2prox. It would be harder to prove a role of the SA1D2prox sequence for in vivo replication via the effect of the Vif expression level.

The mechanism for HIV-1 mRNA splicing appears to be more complicated than previously thought, especially for vif and vpr mRNA production [17]. Going forward, further studies are required to elucidate the mechanism by which nSNMs found within SA1D2prox alter the vif mRNA/Vif protein expression levels. As we have shown, nSNMs within SA1D2prox alter the D1/A1 splicing product levels and concomitantly inversely change the D1/A2 splicing product levels (this study and [37-39]). In fact, we have confirmed the fluctuation in the Vif expression level for pro-viral clones that carry various chimeric vif sequences containing SA2 and SD3 between subtype B (NL4-3) and subtype $\mathrm{C}$ (IndieC) [40]. Given the long-range interaction between splicing sites [62], nucleotide sequences that affect the vif mRNA/Vif protein expression level would be present in various areas on the HIV-1 genome. Even if so, the results using the SA1D2prox sequence from patients suggested the possibility that the Vif expression level can be estimated based on the data for changes in the vif mRNA/Vif protein expression levels by individual mutations (Figures 9 and 10). Once we can comprehensively identify mutations that affect the Vif expression levels, we may be able to predict the expression level just by looking at the sequence. Collectively, clarifying the significance of individual mutations experimentally identified is crucial for understanding viral replication, adaptation, and pathogenesis. 
Author Contributions: Conceptualization, M.N.; investigation, T.K., N.D., M.T., K.W., H.Y., S.N. and M.N.; writing-original draft preparation, A.A. and M.N.; writing-review and editing, A.A. and M.N.; supervision, M.N.; funding acquisition, T.K. and M.N. All authors have read and agreed to the published version of the manuscript.

Funding: This work was supported in part by a Grant-in-Aid for Scientific Research (C) from JSPS to M.N. (JSPS KAKENHI JP21K07042) and grants from Takeda Science Foundation to T.K. and M.N.

Acknowledgments: We thank Yayoi Takada for experimental assistance. We also thank Kazuko Yoshida and Kyoko Inui for editorial assistance. We appreciate the Support Center for Advanced Medical Sciences, Institute of Biomedical Sciences, and Tokushima University Graduate School for experimental facilities and technical assistance.

Conflicts of Interest: The authors declare no conflict of interest.

\section{References}

1. Malim, M.H.; Emerman, M. HIV-1 accessory proteins-Ensuring viral survival in a hostile environment. Cell Host Microbe 2008, 3, 388-398. [CrossRef]

2. Harris, R.S.; Hultquist, J.F.; Evans, D.T. The restriction factors of human immunodeficiency virus. J. Biol. Chem. 2012, 287, 40875-40883. [CrossRef]

3. Malim, M.H.; Bieniasz, P.D. HIV restriction factors and mechanisms of evasion. Cold Spring Harb. Perspect. Med. 2012, 2, a006940. [CrossRef] [PubMed]

4. Aydin, H.; Taylor, M.W.; Lee, J.E. Structure-guided analysis of the human APOBEC3-HIV restrictome. Structure 2014, 22, 668-684. [CrossRef] [PubMed]

5. Desimmie, B.A.; Delviks-Frankenberrry, K.A.; Burdick, R.C.; Qi, D.; Izumi, T.; Pathak, V.K. Multiple APOBEC3 restriction factors for HIV-1 and one Vif to rule them all. J. Mol. Biol. 2014, 426, 1220-1245. [CrossRef]

6. Feng, Y.; Baig, T.T.; Love, R.P.; Chelico, L. Suppression of APOBEC3-mediated restriction of HIV-1 by Vif. Front. Microbiol. 2014, 5, 450. [CrossRef] [PubMed]

7. Okada, A.; Iwatani, Y. APOBEC3G-mediated G-to-A hypermutation of the HIV-1 genome: The missing link in antiviral molecular mechanisms. Front. Microbiol. 2016, 7, 2027. [CrossRef] [PubMed]

8. Stupfler, B.; Verriez, C.; Gallois-Montbrun, S.; Marquet, R.; Paillart, J.C. Degradation-independent inhibition of APOBEC3G by the HIV-1 Vif protein. Viruses 2021, 13, 617. [CrossRef] [PubMed]

9. Xu, H.; Chertova, E.; Chen, J.; Ott, D.E.; Roser, J.D.; Hu, W.S.; Pathak, V.K. Stoichiometry of the antiviral protein APOBEC3G in HIV-1 virions. Virology 2007, 360, 247-256. [CrossRef] [PubMed]

10. Browne, E.P.; Allers, C.; Landau, N.R. Restriction of HIV-1 by APOBEC3G is cytidine deaminase-dependent. Virology 2009, 387, 313-321. [CrossRef]

11. Schwartz, S.; Felber, B.K.; Benko, D.M.; Fenyö, E.M.; Pavlakis, G.N. Cloning and functional analysis of multiply spliced mRNA species of human immunodeficiency virus type 1. J. Virol. 1990, 64, 2519-2529. [CrossRef] [PubMed]

12. Purcell, D.F.J.; Martin, M.A. Alternative splicing of human immunodeficiency virus type 1 mRNA modulates viral protein expression, replication, and infectivity. J. Virol. 1993, 67, 6365-6378. [CrossRef]

13. Amendt, B.A.; Si, Z.H.; Stoltzfus, C.M. Presence of exon splicing silencers within human immunodeficiency virus type 1 tat exon 2 and tat-rev exon 3: Evidence for inhibition mediated by cellular factors. Mol. Cell. Biol. 1995, 15, 4606-4615. [CrossRef] [PubMed]

14. Emery, A.; Zhou, S.; Pollom, E.; Swanstrom, R. Characterizing HIV-1 splicing by using next-generation sequencing. J. Virol. 2017, 91, e02515-16. [CrossRef] [PubMed]

15. Bevilacqua, P.C.; Ritchey, L.E.; Su, Z.; Assmann, S.M. Genome-wide analysis of RNA secondary structure. Annu. Rev. Genet. 2016, 50, 235-266. [CrossRef]

16. Sertznig, H.; Hillebrand, F.; Erkelenz, S.; Schaal, H.; Widera, M. Behind the scenes of HIV-1 replication: Alternative splicing as the dependency factor on the quiet. Virology 2018, 516, 176-188. [CrossRef]

17. Emery, A.; Swanstrom, R. HIV-1: To splice or not to splice, that is the question. Viruses 2021, 13, 181. [CrossRef]

18. Kammler, S.; Otte, M.; Hauber, I.; Kjems, J.; Hauber, J.; Schaal, H. The strength of the HIV-1 30 splice sites affects Rev function. Retrovirology 2006, 3, 89. [CrossRef] [PubMed]

19. Exline, C.M.; Feng, Z.; Stoltzfus, C.M. Negative and positive mRNA splicing elements act competitively to regulate human immunodeficiency virus type 1 vif gene expression. J. Virol. 2008, 82, 3921-3931. [CrossRef] [PubMed]

20. Brillen, A.L.; Walotka, L.; Hillebrand, F.; Müller, L.; Widera, M.; Theiss, S.; Schaal, H. Analysis of competing HIV-1 splice donor sites uncovers a tight cluster of splicing regulatory elements within exon 2/2b. J. Virol. 2017, 91, e00389-17. [CrossRef]

21. Widera, M.; Erkelenz, S.; Hillebrand, F.; Krikoni, A.; Widera, D.; Kaisers, W.; Deenen, R.; Gombert, M.; Dellen, R.; Pfeiffer, T.; et al. An intronic G run within HIV-1 intron 2 is critical for splicing regulation of vif mRNA. J. Virol. 2013, 87, 2707-2720. [CrossRef]

22. Mansky, L.M.; Temin, H.M. Lower in vivo mutation rate of human immunodeficiency virus type 1 than that predicted from the fidelity of purified reverse transcriptase. J. Virol. 1995, 69, 5087-5094. [CrossRef] [PubMed] 
23. Levy, D.N.; Aldrovandi, G.M.; Kutsch, O.; Shaw, G.M. Dynamics of HIV-1 recombination in its natural target cells. Proc. Natl. Acad. Sci. USA 2004, 101, 4204-4209. [CrossRef] [PubMed]

24. Coffin, J.M. HIV population dynamics in vivo: Implications for genetic variation, pathogenesis, and therapy. Science 1995, 267, 483-489. [CrossRef] [PubMed]

25. Malim, M.H.; Emerman, M. HIV-1 sequence variation: Drift, shift, and attenuation. Cell 2001, 104, 469-472. [CrossRef]

26. van Opijnen, T.; Berkhout, B. The host environment drives HIV-1 fitness. Rev. Med. Virol. 2005, 15, 219-233. [CrossRef]

27. Carlson, J.M.; Le, A.Q.; Shahid, A.; Brumme, Z.L. HIV-1 adaptation to HLA: A window into virus-host immune interactions. Trends Microbiol. 2015, 23, 212-224. [CrossRef] [PubMed]

28. Nijhuis, M.; van Maarseveen, N.M.; Lastere, S.; Schipper, P.; Coakley, E.; Glass, B.; Rovenska, M.; de Jong, D.; Chappey, C.; Goedegebuure, I.W.; et al. A novel substrate-based HIV-1 protease inhibitor drug resistance mechanism. PLoS Med. 2007, 4, e36. [CrossRef]

29. Parry, C.M.; Kolli, M.; Myers, R.E.; Cane, P.A.; Schiffer, C.; Pillay, D. Three residues in HIV-1 matrix contribute to protease inhibitor susceptibility and replication capacity. Antimicrob. Agents Chemother. 2011, 55, 1106-1113. [CrossRef]

30. Rabi, S.A.; Laird, G.M.; Durand, C.M.; Laskey, S.; Shan, L.; Bailey, J.R.; Chioma, S.; Moore, R.D.; Siliciano, R.F. Multi-step inhibition explains HIV-1 protease inhibitor pharmacodynamics and resistance. J. Clin. Investig. 2013, 123, 3848-3860. [CrossRef]

31. Blanch-Lombarte, O.; Santos, J.R.; Peña, R.; Jiménez-Moyano, E.; Clotet, B.; Paredes, R.; Prado, J.G. HIV-1 Gag mutations alone are sufficient to reduce darunavir susceptibility during virological failure to boosted PI therapy. J. Antimicrob. Chemother. 2020, 75, 2535-2546. [CrossRef]

32. Hikichi, Y.; Van Duyne, R.; Pham, P.; Groebner, J.L.; Wiegand, A.; Mellors, J.W.; Kearney, M.F.; Freed, E.O. Mechanistic analysis of the broad antiretroviral resistance conferred by HIV-1 envelope glycoprotein mutations. mBio 2021, 12, e03134-20. [CrossRef]

33. Ikeda, T.; Symeonides, M.; Albin, J.S.; Li, M.; Thali, M.; Harris, R.S. HIV-1 adaptation studies reveal a novel Env-mediated homeostasis mechanism for evading lethal hypermutation by APOBEC3G. PLoS Pathog. 2018, 14, e1007010. [CrossRef]

34. Haché, G.; Abbink, T.E.; Berkhout, B.; Harris, R.S. Optimal translation initiation enables Vif-deficient human immunodeficiency virus type 1 to escape restriction by APOBEC3G. J. Virol. 2009, 83, 5956-5960. [CrossRef]

35. Miyagi, E.; Kao, S.; Fumitaka, M.; Buckler-White, A.; Plishka, R.; Strebel, K. Long-term passage of Vif-null HIV-1 in CD4 ${ }^{+}$T cells expressing sub-lethal levels of APOBEC proteins fails to develop APOBEC resistance. Virology 2017, 504, 1-11. [CrossRef]

36. Nomaguchi, M.; Doi, N.; Fujiwara, S.; Saito, A.; Akari, H.; Nakayama, E.E.; Shioda, T.; Yokoyama, M.; Sato, H.; Adachi, A. Systemic biological analysis of the mutations in two distinct HIV-1mt genomes occurred during replication in macaque cells. Microbes Infect. 2013, 15, 319-328. [CrossRef] [PubMed]

37. Nomaguchi, M.; Miyake, A.; Doi, N.; Fujiwara, S.; Miyazaki, Y.; Tsunetsugu-Yokota, Y.; Yokoyama, M.; Sato, H.; Masuda, T.; Adachi, A. Natural single-nucleotide polymorphisms in the $3^{\prime}$ region of the HIV-1 pol gene modulate viral replication ability. $J$. Virol. 2014, 88, 4145-4160. [CrossRef]

38. Nomaguchi, M.; Doi, N.; Sakai, Y.; Ode, H.; Iwatani, Y.; Ueno, T.; Matsumoto, Y.; Miyazaki, Y.; Masuda, T.; Adachi, A. Natural single-nucleotide variations in the HIV-1 genomic SA1prox region can alter viral replication ability by regulating Vif expression levels. J. Virol. 2016, 90, 4563-4578. [CrossRef]

39. Nomaguchi, M.; Doi, N.; Yoshida, T.; Koma, T.; Adachi, S.; Ode, H.; Iwatani, Y.; Yokoyama, M.; Sato, H.; Adachi, A. Production of HIV-1 vif mRNA is modulated by natural nucleotide variations and SLSA1 RNA structure in SA1D2prox genomic region. Front. Microbiol. 2017, 8, 2542. [CrossRef] [PubMed]

40. Doi, N.; Koma, T.; Adachi, A.; Nomaguchi, M. Expression level of HIV-1 Vif can be fluctuated by natural nucleotide variations in the vif-coding and regulatory SA1D2prox sequences of the proviral genome. Front. Microbiol. 2019, 10, 2758. [CrossRef] [PubMed]

41. Adachi, A.; Gendelman, H.E.; Koenig, S.; Folks, T.; Willey, R.; Rabson, A.; Martin, M.A. Production of acquired immunodeficiency syndrome-associated retrovirus in human and nonhuman cells transfected with an infectious molecular clone. J. Virol. 1986, 59, 284-291. [CrossRef]

42. Adachi, A.; Ono, N.; Sakai, H.; Ogawa, K.; Shibata, R.; Kiyomasu, T.; Masuike, H.; Ueda, S. Generation and characterization of the human immunodeficiency virus type 1 mutants. Arch. Virol. 1991, 117, 45-58. [CrossRef]

43. Yamashita, T.; Kamada, K.; Hatcho, K.; Adachi, A.; Nomaguchi, M. Identification of amino acid residues in HIV-1 Vif critical for binding and exclusion of APOBEC3G/F. Microbes Infect. 2008, 10, 1142-1149. [CrossRef]

44. Yamashita, T.; Nomaguchi, M.; Miyake, A.; Uchiyama, T.; Adachi, A. Status of APOBEC3G/F in cells and progeny virions modulated by Vif determines HIV-1 infectivity. Microbes Infect. 2010, 12, 166-171. [CrossRef]

45. Lebkowski, J.S.; Clancy, S.; Calos, M.P. Simian virus 40 replication in adenovirus-transformed human cells antagonizes gene expression. Nature 1985, 317, 169-171. [CrossRef] [PubMed]

46. Kamada, K.; Igarashi, T.; Martin, M.A.; Khamsri, B.; Hatcho, K.; Yamashita, T.; Fujita, M.; Uchiyama, T.; Adachi, A. Generation of HIV-1 derivatives that productively infect macaque monkey lymphoid cells. Proc. Natl. Acad. Sci. USA 2006, 103, 16959-16964. [CrossRef] [PubMed]

47. Nomaguchi, M.; Yokoyama, M.; Kono, K.; Nakayama, E.E.; Shioda, T.; Doi, N.; Fujiwara, S.; Saito, A.; Akari, H.; Miyakawa, K.; et al. Generation of rhesus macaque-tropic HIV-1 clones that are resistant to major anti-HIV-1 restriction factors. J. Virol. 2013, 87, 11447-11461. [CrossRef] 
48. Willey, R.L.; Smith, D.H.; Lasky, L.A.; Theodore, T.S.; Earl, P.L.; Moss, B.; Capon, D.J.; Martin, M.A. In vitro mutagenesis identifies a region within the envelope gene of the human immunodeficiency virus that is critical for infectivity. J. Virol. 1988, 62, 139-147. [CrossRef] [PubMed]

49. Akari, H.; Fujita, M.; Kao, S.; Khan, M.A.; Shehu-Xhilaga, M.; Adachi, A.; Strebel, K. High level expression of human immunodeficiency virus type-1 Vif inhibits viral infectivity by modulating proteolytic processing of the Gag precursor at the p2/nucleocapsid processing site. J. Biol. Chem. 2004, 279, 12355-12362. [CrossRef] [PubMed]

50. Madsen, J.M.; Stoltzfus, C.M. A suboptimal 5' splice site downstream of HIV-1 splice site A1 is required for unspliced viral mRNA accumulation and efficient virus replication. Retrovirology 2006, 3, 10. [CrossRef]

51. Dang, Y.; Wang, X.; Esselman, W.J.; Zheng, Y.H. Identification of APOBEC3DE as another antiretroviral factor from the human APOBEC family. J. Virol. 2006, 80, 10522-10533. [CrossRef]

52. Piroozmand, A.; Yamamoto, Y.; Khamsri, B.; Fujita, M.; Uchiyama, T.; Adachi, A. Generation and characterization of APOBEC3Gpositive 293T cells for HIV-1 Vif study. J. Med. Investig. 2007, 54, 154-158. [CrossRef]

53. Hu, C.; Saenz, D.T.; Fadel, H.J.; Walker, W.; Peretz, M.; Poeschla, E.M. The HIV-1 central polypurine tract functions as a second line of defense against APOBEC3G/F. J. Virol. 2010, 84, 11981-11993. [CrossRef]

54. Widera, M.; Hillebrand, F.; Erkelenz, S.; Vasudevan, A.A.; Münk, C.; Schaal, H. A functional conserved intronic G run in HIV-1 intron 3 is critical to counteract APOBEC3G-mediated host restriction. Retrovirology 2014, 11, 72. [CrossRef]

55. Ode, H.; Matsuda, M.; Matsuoka, K.; Hachiya, A.; Hattori, J.; Kito, Y.; Yokomaku, Y.; Iwatani, Y.; Sugiura, W. Quasispecies analyses of the HIV-1 near-full-length genome with Illumina MiSeq. Front. Microbiol. 2015, 6, 1258. [CrossRef] [PubMed]

56. Takata, M.A.; Soll, S.J.; Emery, A.; Blanco-Melo, D.; Swanstrom, R.; Bieniasz, P.D. Global synonymous mutagenesis identifies cis-acting RNA elements that regulate HIV-1 splicing and replication. PLoS Pathog. 2018, 14, e1006824. [CrossRef]

57. Pagani, F.; Raponi, M.; Baralle, F.E. Synonymous mutations in CFTR exon 12 affect splicing and are not neutral in evolution. Proc. Natl. Acad. Sci. USA 2005, 102, 6368-6372. [CrossRef] [PubMed]

58. Presnyak, V.; Alhusaini, N.; Chen, Y.H.; Martin, S.; Morris, N.; Kline, N.; Olson, S.; Weinberg, D.; Baker, K.E.; Graveley, B.R.; et al. Codon optimality is a major determinant of mRNA stability. Cell 2015, 160, 1111-1124. [CrossRef]

59. Plotkin, J.B.; Kudla, G. Synonymous but not the same: The causes and consequences of codon bias. Nat. Rev. Genet. 2011, 12, 32-42. [CrossRef] [PubMed]

60. Jordan-Paiz, A.; Franco, S.; Martínez, M.A. Impact of synonymous genome recoding on the HIV life cycle. Front. Microbiol. 2021, 12, 606087. [CrossRef]

61. Moris, A.; Murray, S.; Cardinaud, S. AID and APOBECs span the gap between innate and adaptive immunity. Front. Microbiol. 2014, 5, 534. [CrossRef]

62. Pollom, E.; Dang, K.K.; Potter, E.L.; Gorelick, R.J.; Burch, C.L.; Weeks, K.M.; Swanstrom, R. Comparison of SIV and HIV-1 genomic RNA structures reveals impact of sequence evolution on conserved and non-conserved structural motifs. PLoS Pathog. 2013, 9, e1003294. [CrossRef] 